\title{
Republic of Tajikistan: Staff-Monitored Program
}

This paper on the staff-monitored program for Republic of Tajikistan was prepared by a staff team of the International Monetary Fund as background documentation for the periodic consultation with the member country. It is based on information available at the time it was completed on June 10, 2008. The views expressed in this document are those of the staff team and do not necessarily reflect the views of the government of the Republic of Tajikistan or the Executive Board of the IMF.

The policy of publication of staff reports and other documents by the IMF allows for the deletion of market-sensitive information.

Copies of this report are available to the public from

International Monetary Fund $\bullet$ Publication Services

700 19th Street, N.W. • Washington, D.C. 20431

Telephone: (202) 623-7430 • Telefax: (202) 623-7201

E-mail: publications@imf.org • Internet: http://www.imf.org

Price: $\$ 18.00$ a copy

\section{International Monetary Fund Washington, D.C.}





\title{
INTERNATIONAL MONETARY FUND
}

\section{REPUBLIC OF TAJIKISTAN}

\section{Staff-Monitored Program}

\author{
Prepared by the Middle East and Central Asia Department \\ (In consultation with other departments) \\ Approved by David Owen and Mark Plant
}

June 10, 2008

Mission dates: The mission team visited Dushanbe April 17-30, 2008.

Team: C. Piñerúa (head), S. Çakır, F. Alturki, S. Vtyurina (all MCD), A.Unigovskaya (PDR), M. Cortes (MCM), and L. Moers (resident representative). Mr. Owen (MCD) and Mr. Moser (OED) participated in the final discussions.

Key Tajik officials: President Rakhmon, State Economic Advisor Davlatov, Minister of Finance Nadjmuddinov, and National Bank of Tajikistan Chairman Rakhimzoda.

Fund relations: The last Article IV consultation was concluded on March 28, 2007. On March 5, 2008, the Executive Board met to discuss the misreporting episode under the last PRGF arrangement. They supported the Managing Director's recommendation that Tajikistan repay early the three noncomplying disbursements (amounting to SDR 29.4 million) that were not discharged under MDRI relief, with the first payment of SDR 4.9 million expected on September 5, 2008 and equal monthly payments thereafter.

Staff-Monitored Program: In the Memorandum of Economic and Financial Polices attached to the President's Letter of Intent (LoI), the authorities have indicated their intention to implement an economic program covering the period through end-2008, including prior actions, structural benchmarks, and quarterly indicative targets.

Outreach: The mission met with local representatives of the Asian Development Bank, the World Bank, EBRD, EU, UNDP, and briefed members of the diplomatic community. There were two press briefings during the mission. 


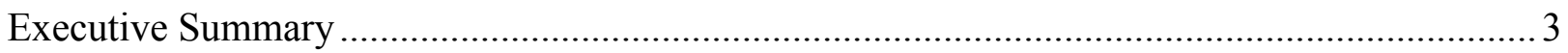

I. Introduction and Background ………....................................................................... 4

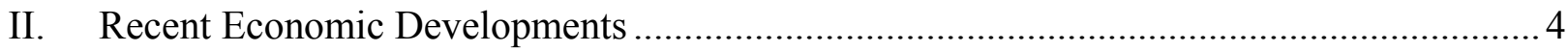

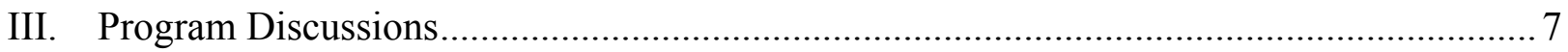

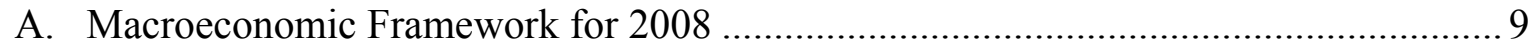

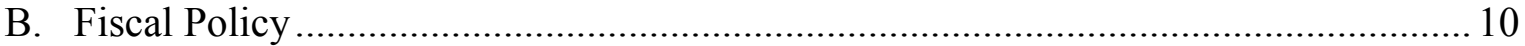

C. Monetary and Exchange Rate Policies................................................................... 10

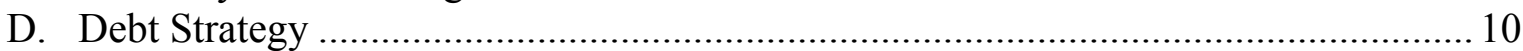

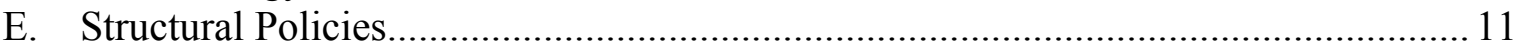

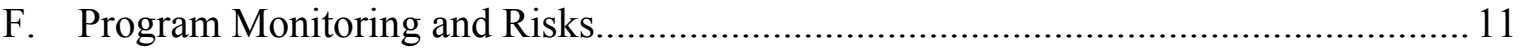

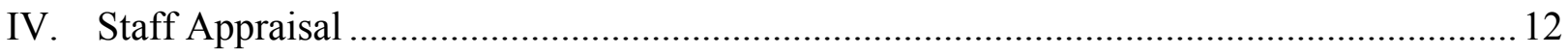

Boxes

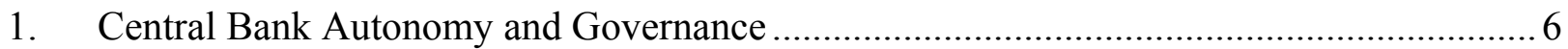

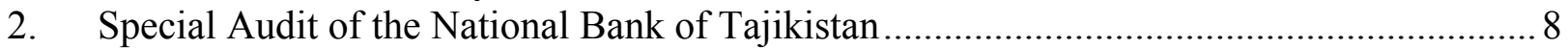

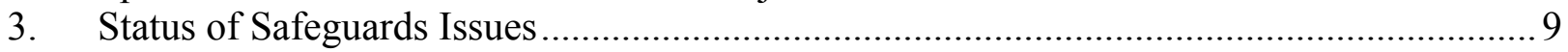

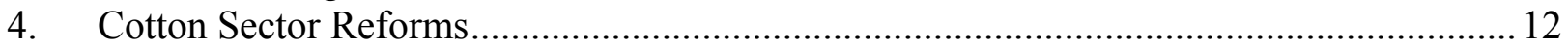

Figure

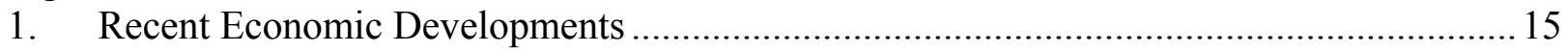

Tables

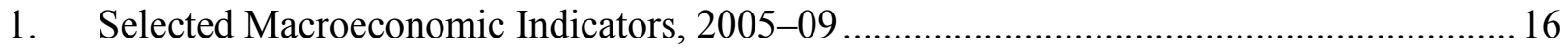

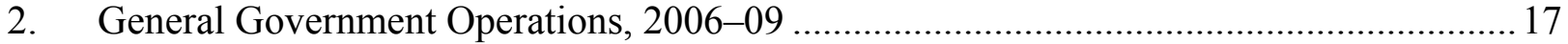

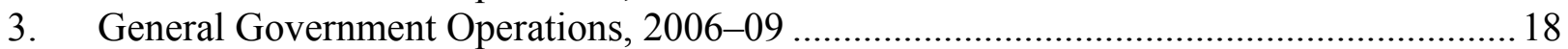

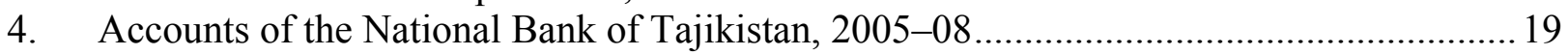

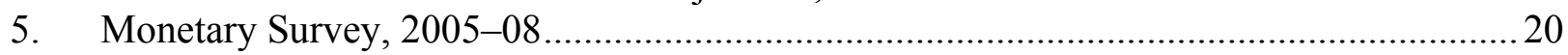

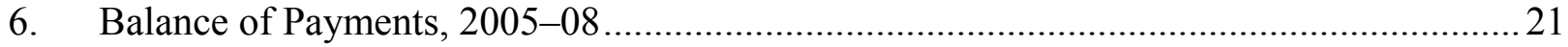

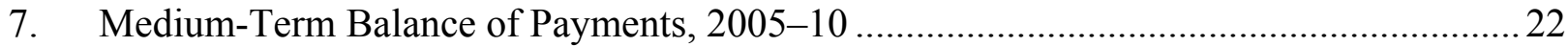

8. Medium-Term Macroeconomic Projections, 2005-12 ……………………………........ 23

Attachments

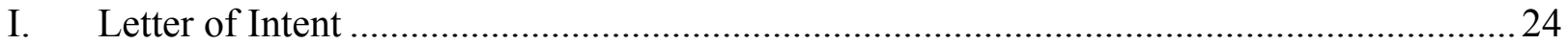

II. Memorandum of Economic and Financial Policies .........................................................2 25

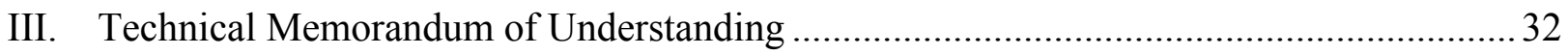




\section{EXECUTIVE SUMMARY}

Recent Developments. Severe weather conditions and related energy shortages early in the year led to a significant slowdown in growth, with real GDP expanding by only 3.2 percent in the first quarter of 2008 relative to the same period in 2007 (compared to 7.8 percent for 2007 as a whole). The inflation rate as of end-March 2008, at 20.3 percent on a 12-month basis, remained essentially unchanged from its end-December 2007 level. Perhaps reacting to the economic hardship, remittances surged in the first quarter, which allowed the National Bank of Tajikistan (NBT) to accumulate reserves and led to a significant overperformance in consumption-related tax revenue collections.

The Staff-Monitored Program (SMP). The program envisages a slowdown in growth in 2008 (to 5 percent), largely reflecting the impact of the harsh winter, and targets a reduction in inflation to 15 percent. The program targets a balanced budget (moving from a small surplus in 2007), which will allow for a real increase in social outlays and transparent financing of the cotton sector. The monetary policy framework relies on a reserve money target and a managed float exchange rate regime. The program's debt management framework includes a zero ceiling on the contracting of nonconcessional debt and the establishment of a contingent liability reporting regime to cover state-owned enterprises and the NBT.

\section{Measures to address governance and management problems at the NBT are central to} the SMP. Program conditionality in this area is linked to the conduct of a special audit of the NBT. Terms of reference (ToR) for the audit were finalized and agreed with staff as a prior action. On May 14, the authorities forwarded the ToR to the potential specialist auditor firms seeking bids for the contract (also a prior action). The program also includes measures to strengthen the autonomy and governance of the NBT, including through the passage of appropriate amendments to the central bank law. In addition, the SMP attempts to address long-standing shortcomings in the governance and monitoring of the largest state-owned enterprises (SOEs), including through the undertaking of external audits of the two largest SOEs by reputable international firms.

Key Risk. The main risk to the success of the program is the possibility of delays in completing the special audit of the NBT. This could arise because of the complexity and lack of adequate documentation of cotton sector transactions; the authorities' full cooperation with the auditors will therefore be essential. The first review of the program (October 2008) will focus on assessing progress in this area. 


\section{INTRODUCTION AND BACKGROUND}

\section{On March 5, 2008, the Executive Board met to discuss the recent misreporting} episode. ${ }^{1}$ Directors agreed that the provision of inaccurate data to the Fund-involving the level of international reserves and net domestic assets of the National Bank of Tajikistan (NBT), and the issuance of directed credit by the NBT-over the past six years had seriously undermined the credibility of the authorities with IFIs and other donors. They supported the Managing Director's recommendation that Tajikistan repay early the three noncomplying disbursements (amounting to SDR 29.4 million) that were not discharged under MDRI relief. Moreover, Directors noted that the authorities would need to take decisive actions to restore credibility and resume the path of policy reforms, with most supporting the idea of putting in place a Staff-Monitored Program (SMP) to run in parallel with a proposed special audit of the NBT. The Executive Board did not require any further remedial action be taken by the Tajik authorities with respect to the breach of obligations under Article VIII, Section 5 as a result of the misreporting.

\section{RECENT ECONOMIC DEVELOPMENTS}

2. While growth accelerated in 2007, inflation also surged to its highest level since 2003. Although real GDP grew by 7.8 percent in 2007 (compared to 7 percent in 2006), driven mainly by remittance-financed demand in the services and construction sectors, it slowed sharply in the first quarter of 2008 (to 3.2 percent year-on-year) as the country was hit by harsh winter conditions and related shortages in electricity supplies. ${ }^{2}$ As of end-March 2008 , inflation remained stubbornly high at about 20 percent, mostly reflecting a surge in international food and energy prices, but also a loose monetary policy stance in 2007 . More recently, reflecting a surge in remittances inflows and tighter financial policies in early 2008 , the somoni has come under pressure to appreciate, allowing the NBT to replenish its reserves to some degree. ${ }^{3}$ Nevertheless, the reserves of the NBT are now largely encumbered (see paragraph 5).

3. Budget targets were met with some margin in 2007. With a strong tax revenue performance - mostly reflecting rapid import growth and higher than planned inflation - and spending in line with the budget, the budget recorded a surplus of about $1 \frac{1}{2}$ percent of GDP in 2007 (excluding the externally financed Public Investment Program (PIP)), compared to a

\footnotetext{
${ }^{1}$ See Republic of Tajikistan-Report on Noncomplying Disbursements and Breach of Obligations Under Article VIII, Section 5 (EBS/08/21).

${ }^{2}$ On February 18, the UN appealed for $\$ 25$ million to meet urgent humanitarian needs in Tajikistan.

${ }^{3}$ MCM recently reclassified Tajikistan's exchange rate framework as a conventional peg from a managed float. In this connection, the mission held a seminar on the implications of the 2007 Surveillance Decision.
} 
deficit target of 1 percent of GDP. ${ }^{4}$ While the fiscal overperformance led to a significant accumulation of government deposits at the NBT, reserve money growth remained high as the NBT issued large amounts of credit to the cotton sector.

4. The strong revenue performance appears to have spilled over into early $\mathbf{2 0 0 8 \text { , }}$ mostly reflecting very high consumption-related tax revenue collections linked to remittances inflows. The 2008 budget approved last November is moderately expansionary, to allow for more transparent financing of the cotton sector, support payments to farmers, and a 40 percent increase in the wage bill. While the original aim of the authorities was to target a deficit of $1 / 2$ percent of GDP (excluding the PIP), the strong revenue performance so far could allow for a tighter stance.

\section{The misreporting episode shed light on a number of serious external}

vulnerabilities and structural weaknesses. As part of the misreporting assessment process, staff learned from the authorities that, in order to obtain financing for the cotton sector, the NBT issued written guarantees to foreign creditors and also pledged its reserves as collateral by depositing its reserves at the creditor banks. As of March 2008, the NBT had issued about $\$ 70$ million in the form of written guarantees, while total NBT pledges amounted to about $\$ 240$ million. This compares to gross foreign assets, as reported to the Fund, of about $\$ 385$ million. Therefore, import reserve coverage is now very low, at about half a month of imports. Moreover, these previously unreported debt obligations ( $\$ 310$ million) carry a short maturity (between three and six months) and thus require frequent rollovers. ${ }^{56}$

\section{Progress in structural reform has been uneven:}

- While the banking sector appears well-capitalized and liquid, last year's FSAP missions noted that the supervisory framework is highly susceptible to political influence. Moreover, even before misreporting came to light, the FSAP team stressed that the NBT was insolvent and that its autonomy and governance required significant strengthening (Box 1).

\footnotetext{
${ }^{4}$ Project disbursements from China, the largest PIP component, amounted to $\$ 217$ million (6 percent of GDP) in 2007, somewhat behind schedule due to reported bottlenecks in the disbursement process.

${ }^{5}$ All of these obligations have been contracted by KreditInvest, a private nonbank financial institution created in 2003 to manage bad assets previously held by the Agricultural Bank (Agroinvestbank).

${ }^{6}$ The external public and publicly guaranteed debt (excluding NBT pledges) stood at \$1,231 million at end2007 , or 33 percent of GDP.
} 


\section{Box 1. Tajikistan: Central Bank Autonomy and Governance}

The autonomy and governance of the National Bank of Tajikistan (NBT) require considerable strengthening, including in the areas of:

- Financial autonomy. The NBT should be appropriately capitalized to ensure its financial independence, but not before its involvement in commercial activities stops. The NBT is currently insolvent, largely the result of undertaking quasi-fiscal obligations, which could grow if the cotton sector defaults on its external obligations.

- Terms of appointment and removal of board members. The NBT chairman and his deputies should be appointed for fixed terms, which should not coincide with the electoral cycle. Moreover, the reasons for their dismissal should be explicitly outlined in the law.

- Conflict of interest. Clear provisions covering conflicts of interest should be introduced in the law. Specifically, while the central bank law does state that board members and other NBT employees may not represent any business interest that conflicts with their official obligations, there are no provisions on what constitutes conflict of interest, how the central bank monitors possible conflicts of interest of officials and staff, and which sanctions should be applied in the case of violations.

- Disclosure of beneficial ownership. The law should require official identification and disclosure of beneficial owners of registered companies, including financial institutions. Lack of public disclosure of significant shareholders of financial institutions and of legal definitions for connected business interests and beneficial ownership are key obstacles to good governance, the prevention of conflicts of interest, and implementation of prudential norms (e.g.; limits on connected party lending).

- There has been some progress in improving the finances of the energy sector, but a large agenda remains. Gas tariffs are currently at cost recovery levels, and gas and electricity collection rates for most groups of consumers have improved.

Nevertheless, electricity tariffs, which have been adjusted considerably in the last two years, are still below levels that would make the electricity sector financially viable, leaving the state-owned electricity company, Barki Tajik, in an uncertain financial situation. ${ }^{7}$ Moreover, regulatory weaknesses in the electricity sector continue to undermine its significant potential.

- The recent misreporting episode exposed fundamental shortcomings in land reform efforts. Even though most state and collective farms have been dismantled and a significant number of land share certificates issued to private farmers, restructured farms are still subject to state control over production and harvesting decisions, particularly in the cotton sector. Under the current system, most farmers are forced to

\footnotetext{
${ }^{7}$ The recent weather-related problems exposed years of serious mismanagement of the sector, including underinvestment and lack of appropriate maintenance.
} 
grow cotton rather than other crops. Moreover, financing for the cotton sector is provided by politically powerful "investors" that also control the supply of inputs and own most of the cotton ginneries. As a stop-gap measure, in January 2008 the government put in place a cotton financing mechanism that uses budget funds (130 million somoni) to be onlent to farmers through commercial banks with low margins. ${ }^{8}$

\section{While budget reporting has improved significantly, efforts to monitor and} enhance the corporate governance of state-owned enterprises are still lacking. As noted in the 2006 fiscal ROSC, the operations of key state-owned enterprises remain beyond the control of the ministry of finance, their owner. Currently, the ministry of finance lacks the appropriate supervisory framework to monitor the financial operations of even the largest state-owned companies. Moreover, these enterprises have no public disclosure requirements and their financial statements are not audited by any of the major internationally recognized auditing firms. Most worrisome is that the financial operations of the aluminum company (Talco), the largest SOE in Tajikistan, remain nontransparent.

\section{Program Discussions}

8. The authorities agreed with staff that the main objectives of the SMP should be twofold:

- To reestablish their credibility by addressing the governance and management problems at the NBT. Key policy measures in this area relate to the process of conducting a special audit of the NBT and other involved parties agreed to by President Rakhmon in his letter to the Managing Director dated February 2, 2008 (Box 2). Draft terms of reference for the audit were prepared in consultation with FIN (Box 3 ) and the authorities agreed them with the mission. The program also includes measures to strengthen the autonomy and governance of the NBT outlined in the recent FSAP aide memoire, including through the introduction of appropriate amendments to the central bank law. ${ }^{9}$ In addition, in order to improve transparency in other government operations, the authorities agreed to address long-standing shortcomings in the corporate governance and monitoring of the largest SOEs.

\footnotetext{
${ }^{8}$ Under the scheme, commercial banks bear the credit risks in full and the government may have exerted moral suasion in the setting of lending rates.

${ }^{9}$ The authorities and staff concurred that measures to address the NBT capitalization problem will need to be articulated once the special audit is concluded.
} 


\section{Box 2. Special Audit of the National Bank of Tajikistan}

In order to address the problems that led to the misreporting, a special audit of the National Bank and other involved parties will be conducted by a reputable international auditing firm. The special audit is expected to:

- Assess the effectiveness of the governance structure and control environment at the NBT, with a view to making specific recommendations on steps that should be taken to prevent recurrence of misreporting.

- Verify the revised data reported to the Fund and establish a reliable foundation for the future provision of data to the IMF, and in particular, review the transactions involving balances where information was withheld from both the IMF and the external auditor.

- Make transparent all financial operations of the NBT involving off-balance sheet transactions, such as pledges of international reserves, issuance of guarantees, and extension of domestic credits to Kredit Invest.

- Review the financing transactions in relation to Kredit Invest, confirm the legitimacy of payments to third parties by Kredit Invest, and ascertain Kredit Invest's total liabilities.

- Review the decision-making process at Kredit Invest with a view to determining how financing was allocated to cotton investors and how interest rates and other repayment terms were determined, and verify whether the contractual obligations of the cotton investors to Kredit Invest have been honored.

The audit is expected to last 6-8 months. Interim reports by the auditors will be shared with Fund staff and the audit's key findings will be made public.

- To provide a framework for macroeconomic stability, taking into account existing policy constraints. Given the current low level of reserves and the serious limitations on the flexibility of monetary policy imposed by the insolvency of the NBT, the authorities' macroeconomic policy strategy relies on a prudent fiscal policy stance to restore stability in the short term. To address medium-term concerns about the increased risk of debt problems, the authorities' program puts in place a welldefined and cautious debt strategy. 


\section{Box 3: Status of Safeguards Issues}

A FIN staff team visited Rotterdam and Dushanbe in March to: (i) follow up on the work of PricewaterhouseCoopers (PwC) in their external audits of the NBT, and (ii) determine the terms of reference for the special audit of the NBT and other related parties. Key findings were as follows:

- The previously misreported domestic credits to the cotton sector had largely been financed through issuance of currency. These loans, and the corresponding increase in currency in circulation, were not recorded in the NBT's accounting records made available to the internal and external auditors. As of November 2007, these credits amounted to approximately 800 million somoni (US\$232 million).

- PwC conducted the external audits of the NBT in accordance with International Standards on Auditing, which require the auditor to obtain third-party confirmation of bank balances and any pledges thereon. The existence of pledges and guarantees were not discovered by $\mathrm{PwC}$ because: (i) the relevant information was withheld by the NBT; and (ii) the correspondent banks did not disclose these items during the bank confirmation process. The audit firm has subsequently confirmed these points in writing.

- It is expected that PwC will finalize the 2006 and 2007 annual audits once the special audit of the NBT is concluded.

Prior to consideration of a possible new PRGF arrangement by the Executive Board, the special audit of the NBT would need to be completed. An external audit of the financial statements for the year ended April 30, 2008 would need to be completed without delay. A new safeguards assessment of the NBT will also have to be completed by no later than the first review of such an arrangement.

\section{A. Macroeconomic Framework for 2008}

\section{The program's macroeconomic framework for 2008 envisages a slow down in} growth - to about 5 percent-and targets only a limited reduction in the inflation rate-to 15 percent per annum. The recent weather-related problems are likely to have a negative effect on economic activity and inflation performance during much of 2008. Specifically, the agricultural sector is likely to underperform as it was hit particularly hard by frost. Industrial production growth, after coming to almost a complete halt in the first quarter, is likely to recover with improving energy supplies. Consumer price inflation is targeted to decline, but remain in double digits, largely owing to significant losses in agricultural and food production, further adjustments in electricity and gas tariffs, and the continuing upward trend in international food and fuel prices. Excluding imports of goods and services related to projects financed by China, the current account deficit is expected to stay at around 5 percent of GDP in 2008. The program assumes that the flow of remittances will ease somewhat during the remainder of 2008, but will remain above the 2007 level. Gross reserves are targeted to increase to about $\$ 170$ million, or about 3 weeks of imports. 


\section{B. Fiscal Policy}

10. The proposed program includes a balanced budget objective with a view to securing the inflation goal. While the 2008 budget targets a small fiscal deficit, the authorities agreed with staff that, given the limited flexibility in monetary policy, it would be prudent at this point to make use of the revenue overperformance so far in 2008 and aim for a balanced budget. Given the fiscal overperformance in 2007, aiming for a balanced budget will still allow for an increase in real expenditures, including for higher support payments to farmers for the acquisition of seeds, machinery and fertilizers ( 0.2 percent of GDP); an increase in real wages - from their still very low level — to forestall a persistent exodus of qualified personnel ( 0.5 percent of GDP); and - for the first time - to provide financing to the cotton sector transparently through the budget (0.9 percent of GDP).

\section{While public financial management has improved, there was agreement that} monitoring and corporate governance of state-owned enterprises should be enhanced, along the lines of the recommendations of the 2006 fiscal ROSC. Therefore, the program incorporates steps to address some of the existing shortcomings, including through the creation of a special monitoring unit at the ministry of finance and the tendering for external audits of Talco and Barki Tajik, whose operations could be both a major untapped source of tax revenues and hitherto hidden contingent liabilities.

\section{Monetary and Exchange Rate Policies}

\section{Monetary policy will aim at containing inflation by targeting reserve money} while building up official foreign exchange reserves. Under the program framework, reserve money growth will be targeted to slow sharply, to around the projected growth rate of nominal GDP, implying no change in velocity. Making room for the accumulation of international reserves will limit the scope for a drawdown of government deposits at the NBT consistent with the fiscal program and will leave no room for any further directed credit from the NBT to the private sector. Moreover, given uncertainties about real money demand, the authorities agreed that, should signs emerge that inflation is accelerating, the NBT will respond by adjusting its reserve money target and tightening monetary conditions through an increase in the sales of its own bills. ${ }^{10}$ The NBT also committed to supporting the inflation objective by intervening in the foreign exchange market only to limit excessive short-term fluctuations in the exchange rate and to ensure that targeted reserves gains are achieved.

\section{Debt Strategy}

13. In order to mitigate risks of future debt problems, the program includes a welldefined and cautious debt strategy. This involves:

\footnotetext{
${ }^{10}$ Given the insolvency of the NBT, the cost of mopping up any excess liquidity will eventually need to be borne by the budget as part of the recapitalization of the NBT.
} 
- No new contracting or guaranteeing of nonconcessional debt.

- A commitment that any new concessional foreign debt disbursement over the next three years will be consistent with the government's borrowing plan already approved by Parliament in January 2008.

- Strengthening the authorities' debt data management framework by establishing, with assistance from FAD, a contingent liability reporting regime to cover state-owned enterprises, public institutions and the NBT. ${ }^{11}$

14. This debt strategy should serve to stabilize the external public and publicly guaranteed debt-to-GDP ratio in the medium-term at about 40 percent. The authorities accepted the need for this limit, but stressed that it would could constrain their ability to finance urgently needed investments in new hydroelectric power plants, which are needed to address persistent energy shortages (which were clearly exposed during the recent severe winter) and eventually allow for electricity exports. While mindful of the need to invest in this critical sector, staff urged the authorities to look first into the possibility of attracting nondebt creating inflows (especially foreign direct investment) to meet these financing needs, and encouraged the authorities to continue to pursue the structural reforms needed to make the sector financially viable in the medium term.

\section{E. Structural Policies}

\section{The authorities are taking steps to reinvigorate their cotton sector reform}

efforts. In consultation with the World Bank and the Asian Development Bank, they are in the process of designing and implementing a comprehensive strategy to deal more permanently with the long-standing problems of the sector (Box 4).

\section{F. Program Monitoring and Risks}

16. The program will run from June through December 2008 and comprises quantitative indicative targets and structural benchmarks for end-September and end-December 2008. Reaching understandings with staff on the terms of reference for the special audit and launching the selection process for conducting the special audit were prior actions for seeking approval of the SMP from Fund management. There will be two reviews of the program, one in October 2008 and the other in January 2009. The first review will focus mainly on assessing progress in the conduct of the special audit of the NBT.

\section{The key risk to the program is the possibility of delays in completing the special} audit of the NBT. Avoiding delays will require full cooperation by the authorities with the

\footnotetext{
${ }^{11}$ The mission conducted a seminar on best practices in designing and implementing a medium-term debt management strategy.
} 
auditors, to provide a clear signal of their commitment to increase the transparency in the operations of the NBT. Furthermore, while staff welcomed the increased transparency of the new cotton financing scheme, its current design could entail significant risks to the financial position of the commercial banks involved in it, particularly as they are shouldering all of the credit risks in dealing with already financially overburdened farmers.

\section{Box 4. Cotton Sector Reforms}

Tajikistan's cotton sector has seen a steady decline in productivity and profitability. The incentive structure and lack of efficient regulatory framework allowed cotton processors and traders (investors) to operate as monopsonists in the domestic cotton market and monopolists in providing financing and inputs to farmers. As a result, Tajikistan is faced with two pressing issues: enhancing the viability of the sector and resolving the existing stock of debt.

On March 5, 2007 the Government of Tajikistan issued Decree 111, which addresses distortions in cotton production, including most importantly issues of freedom to farm and ability to use land rights as collateral. Specifically, the decree deals with issues of a) farm debt resolution; b) termination of government interference in cotton production, processing and sale; c) improvements in cotton marketing, pricing and taxation; d) land reform, and e) improved access to rural financing. As regards the outstanding debt stock, they are currently in discussions with Kredit Invest's external creditors seeking to improve the terms of the obligations that were guaranteed by the NBT, including through the extension of maturities and a reduction of interest rates. As part of the process of resolving Kredit Invest, they are also exploring the possibility of swapping some of its external obligations for assets that were used by domestic borrowers (cotton investors) to collateralize their own obligations to Kredit Invest.

The Asian Development Bank (ADB) and World Bank, as well as bilateral donors, continue to provide support for reforms in this sector:

- ADB is currently preparing a Sustainable Cotton Program loan that focuses on market reforms that would ensure that farmers, processors and traders have freedom of choice in production and marketing decisions. This should lead to greater competition and improve the sector's profitability, ultimately addressing the underlying problems that created the debt overhang.

- The World Bank approved a Cotton Sector Recovery Project in 2007 through which the government is receiving advice on debt resolution options.

\section{StafF APPRAisal}

\section{The authorities' commitments under the program signal a willingness to break}

with the past. Specifically, the program provides opportunities to: a) finally create a modern central bank that can conduct effective monetary policy; b) restore the authorities' credibility by cooperating fully with the special audit of the NBT; c) advance cotton reforms by establishing a more transparent financing mechanism; and d) crucially, open a window into the operations of large SOEs that have been a major drain on national resources. 
Nevertheless, while these commitments are welcome and important, the key to the success of the program will be their full implementation. Staff will monitor closely the implementation of not only the letter, but also the spirit of the authorities' commitments under the SMP.

19. It is important that the authorities follow through in their efforts to establish transparency in public institutions. Full cooperation with the specialist auditor will be paramount in this endeavor. The ToR for the special audit have been drafted with the aim of fully exposing those transactions that not only led to the misreporting to the Fund and put the finances of the country in a precarious situation. Nevertheless, getting to the root of the problem by identifying the governance failures that led to this situation is only the beginning. The staff urges the authorities not only to cooperate fully with the auditor-and disclose publicly all the results of the audit — but also to promptly take up the recommendations of the auditor once the work is concluded. Anything less will fail to restore the authorities' credibility in the eyes of the international community. In this connection, unwavering political support for bringing more transparency to the operations of the largest SOEs is also critical.

\section{Maintaining macroeconomic stability will require a prudent fiscal stance,} enhancing the operational independence of the NBT, and a strong commitment to debt sustainability. Given the limited effectiveness of monetary policy, staff believes that the authorities' commitment to a balanced budget is essential, despite some pressing expenditure needs. Should there be an unplanned overperformance in tax revenue collections, the authorities should save those additional resources with a view to improving inflation performance and facilitating the accumulation of international reserves. Furthermore, to strengthen the framework for monetary policy the authorities should press ahead with the reforms of the central bank legislation with urgency. Improving the legislative framework, including by properly defining the concept of conflict of interest and introducing appropriate sanctions, will go a long way toward establishing the NBT's operational independence. At the same time, staff welcomes the planned tightening of monetary conditions, but cautions that this will not be achievable unless the policy of directed credits ends. Finally, while staff fully understands the government's wish to avoid future energy shortages and its need to enhance the country's electricity export potential, it encourages the authorities to focus on providing proper incentives for foreign direct investment to help meet the sector's financing needs, and refrain from contracting new foreign debt obligations for this purpose, particularly at a time when debt ratios are worsening.

\section{While staff welcomes the transparency of the new cotton sector financing} scheme, its possible impact on commercial banks' balance sheets is a concern. The contracts place the costs of loan default exclusively on the banks, in a situation where lending rates do not fully reflect such risks. To safeguard the financial position of the banks involved in this scheme, staff encourages the authorities to introduce, as soon as possible, performance-based management contracts for banks to provide strong incentives for them to collect the outstanding sums without unduly penalizing them for delinquencies. Moreover, to 
enhance the profitability of the cotton sector and thus reduce credit risk for commercial banks, the authorities should vigorously resume the path of cotton sector reform with the assistance of the World Bank and the Asian Development Bank, including by addressing the distortions created by the politically powerful investors and effectively granting farmers the freedom to farm. 
Figure 1. Recent Economic Developments

Growth is slowing down in $2008 \ldots$

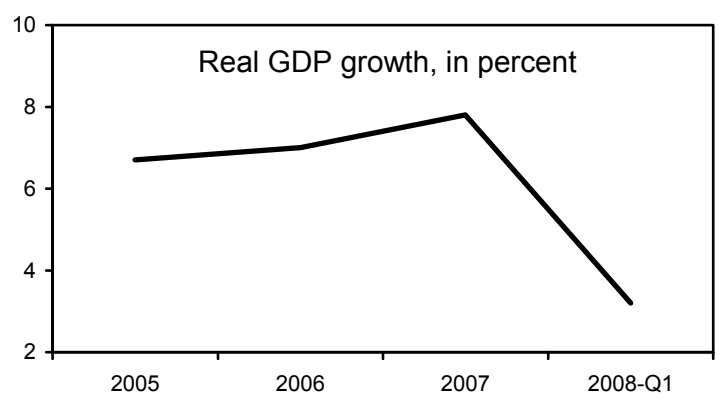

but also a loose monetary stance in $2007 \ldots$

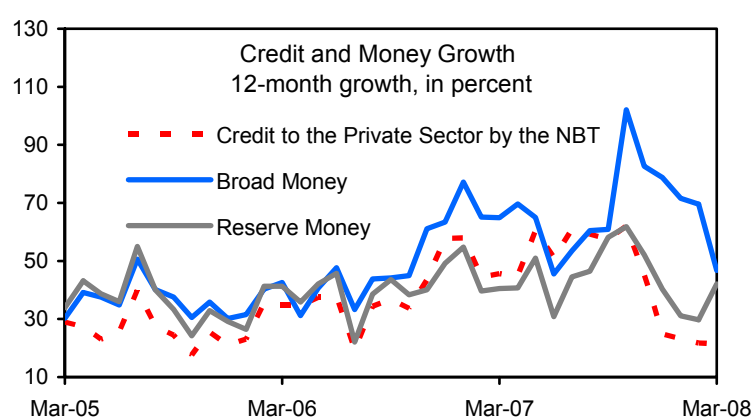

Nevertheless, the NBT's foreign assets are now largely encumbered..

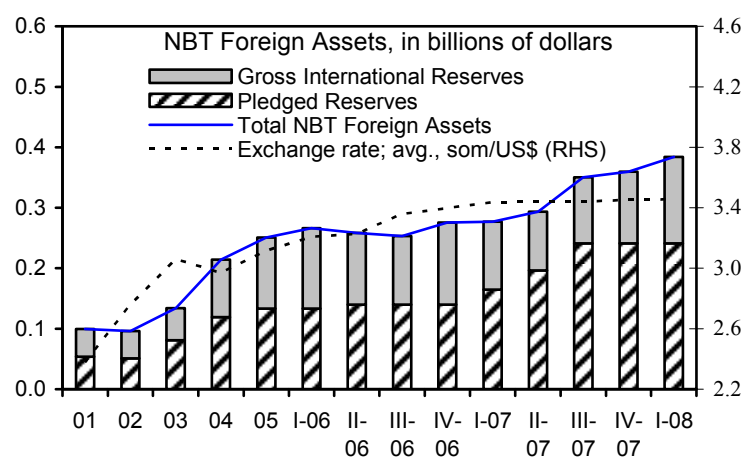

...whereas inflation remains high, mostly reflecting higher food prices...

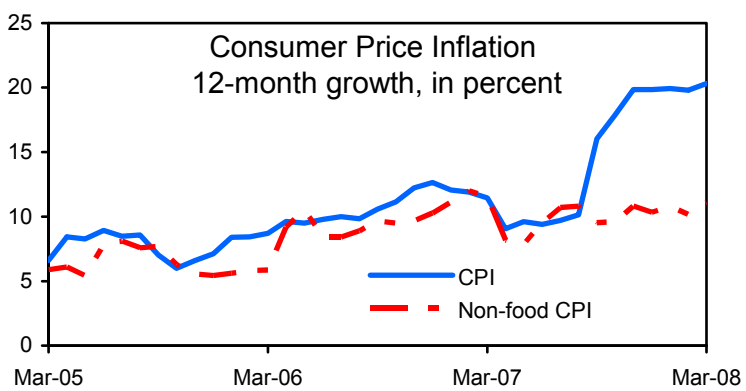

...and despite the accumulation of government deposits at the NBT, reflecting the prudent fiscal stance.

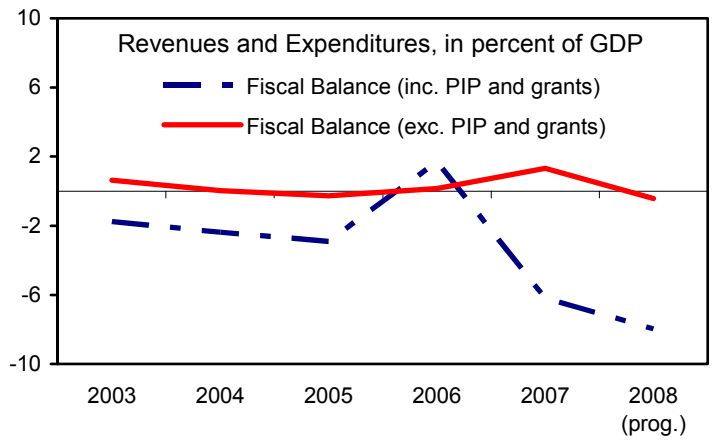

..and debt ratios are worsening.

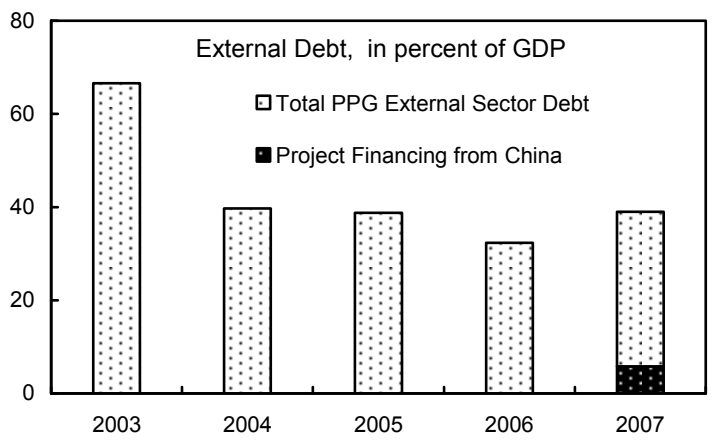

Sources: Tajik authorities; and Fund staff estimates. 
Table 1. Tajikistan: Selected Macroeconomic Indicators, 2005-09

(Quota: SDR 87 million)

(Population: 7 million, 2007)

(Per capita GDP: US\$561; 2007)

\begin{tabular}{|c|c|c|c|c|c|}
\hline & 2005 & 2006 & $\frac{2007}{\text { Est. }}$ & $\frac{2008}{\text { Prog. }}$ & $\frac{2009}{\text { Proj. }}$ \\
\hline \multicolumn{6}{|c|}{ (Annual percent change, unless otherwise indicated) } \\
\hline National accounts & & & & & \\
\hline Nominal GDP (in millions of somoni) & 7,201 & 9,272 & 12,780 & 16,135 & 19,337 \\
\hline Nominal GDP (in millions of dollars) & 2,311 & 2,811 & 3,712 & 4,300 & 4,955 \\
\hline Real GDP & 6.7 & 7.0 & 7.8 & 5.0 & 7.0 \\
\hline CPI inflation (end-of-period) & 7.1 & 12.5 & 19.8 & 15.0 & 9.0 \\
\hline Unemployment rate & 2.1 & 2.3 & 2.3 & 2.3 & 2.3 \\
\hline \multicolumn{6}{|c|}{ (In percent of GDP) } \\
\hline Investment and savings $1 /$ & & & & & \\
\hline Investment & 14.8 & 13.7 & 22.0 & 20.8 & 18.1 \\
\hline Fixed capital investment & 13.8 & 12.8 & 21.1 & 20.0 & 17.3 \\
\hline Government & 7.8 & 6.8 & 14.1 & 14.0 & 11.3 \\
\hline Private & 6.0 & 6.0 & 7.0 & 6.0 & 6.0 \\
\hline Gross national savings & 12.1 & 10.9 & 10.9 & 10.9 & 10.0 \\
\hline Public & 4.9 & 5.0 & 7.9 & 6.1 & 4.8 \\
\hline Private & 7.2 & 9.4 & 2.9 & 4.8 & 5.1 \\
\hline Savings/investment balance & -2.7 & -2.8 & -11.2 & -10.0 & -8.1 \\
\hline \multicolumn{6}{|c|}{ (In percent of GDP) } \\
\hline Revenue and grants $2 /$ & 20.1 & 23.6 & 21.6 & 22.4 & 22.6 \\
\hline Of which: tax revenue & 16.6 & 16.5 & 17.8 & 18.5 & 19.1 \\
\hline Expenditure and net lending & 23.0 & 21.9 & 27.8 & 30.4 & 29.1 \\
\hline Of which: current & 15.1 & 15.0 & 13.6 & 15.4 & 16.9 \\
\hline capital & 7.8 & 6.8 & 14.1 & 14.0 & 11.3 \\
\hline Balance (excluding externally-financed PIP) 2/ & 0.5 & 0.8 & 1.6 & 0.0 & 0.0 \\
\hline Balance (including externally-financed PIP) & -2.9 & 1.7 & -6.2 & -8.0 & -6.4 \\
\hline Domestic financing & 0.3 & -3.3 & -0.7 & 0.8 & 0.0 \\
\hline External financing & 2.6 & 1.6 & 6.9 & 7.2 & 6.4 \\
\hline \multicolumn{6}{|c|}{ (12-month growth in percent of broad money, unless otherwise indicated) } \\
\hline Net foreign assets of the banking sector $3 /$ & -70.3 & 25.1 & 6.4 & 7.7 & -14.9 \\
\hline Net domestic assets of the banking sector 3 / & 129.6 & 87.5 & 112.7 & 40.8 & 58.2 \\
\hline Broad money $3 /$ & 30.2 & 63.4 & 78.8 & 24.7 & 24.8 \\
\hline Reserve money (12-month percent change) & 29.2 & 49.2 & 40.3 & 23.8 & 18.5 \\
\hline Velocity of broad money (eop) & 2.3 & 1.8 & 1.4 & 1.4 & 1.4 \\
\hline Interest rate ( NBT bill rate, in percent) & 7.0 & 7.7 & 7.3 & $\ldots$ & $\ldots$ \\
\hline \multicolumn{6}{|c|}{ (In millions of U.S. dollars, unless otherwise indicated) } \\
\hline External sector $4 /$ & & & & & \\
\hline Exports of goods and services & 601 & 656 & 767 & 856 & 896 \\
\hline Percent change & & 9.1 & 16.9 & 11.6 & 4.8 \\
\hline Imports of goods and services & 1,221 & 1,618 & 2,555 & 3,188 & 3,357 \\
\hline Percent change & & 32.5 & 57.9 & 24.8 & 5.3 \\
\hline Current account balance & -62 & -79 & -414 & -429 & -401 \\
\hline Percent of GDP & -2.7 & -2.8 & -11.2 & -10.0 & -8.1 \\
\hline Trade balance (in percent of GDP) & -26.9 & -35.1 & -45.1 & -50.9 & -46.7 \\
\hline FDI (in percent of GDP) & 2.4 & 2.3 & 4.3 & 4.2 & 3.6 \\
\hline Total public and publicly guaranteed external debt & 897 & 910 & 1,231 & 1,492 & 1,779 \\
\hline Percent of GDP & 38.8 & 32.4 & 33.2 & 34.7 & 35.9 \\
\hline Gross official reserves $5 /$ & 91 & 111 & 107 & 169 & 229 \\
\hline Months of imports (excl. imports financed by loans from China) & 0.9 & 0.7 & 0.5 & 0.8 & 1.0 \\
\hline \multicolumn{6}{|l|}{ Memorandum items: } \\
\hline Nominal effective exchange rate (Index 2000=100) & 58.7 & 54.8 & 50.6 & $\ldots$ & $\ldots$ \\
\hline Real effective exchange rate (Index 2000=100) & 88.9 & 86.5 & 83.9 & $\ldots$ & $\ldots$ \\
\hline Average exchange rate (somoni per dollar) & 3.12 & 3.30 & 3.44 & $\ldots$ & $\ldots$ \\
\hline
\end{tabular}

Sources: Data provided by the Tajikistan authorities; and Fund staff estimates.

1/ Private investment and savings are estimates. Investment includes changes in stocks.

2/ 2006 overall balance excludes the MDRI debt relief that is reflected in grants. PIP expenditure includes investment financed

by loans from China.

$3 /$ Actuals are based on official exchange rates.

4/ Starting from 2005, the export and import figures reflect the transition to tolling arrangement for aluminum exports.

Therefore, the export and import figures are lower than earlier years.

5/ Gross reserves are net of the pledged deposits of the NBT. 
Table 2. Tajikistan: General Government Operations, 2006-09

(In percent of GDP; unless otherwise indicated)

\begin{tabular}{|c|c|c|c|c|c|c|c|c|}
\hline & \multirow{3}{*}{$\begin{array}{r}2006 \\
\text { Act. }\end{array}$} & \multirow{3}{*}{$\begin{array}{r}2007 \\
\text { Est. }\end{array}$} & \multicolumn{5}{|c|}{2008} & \multirow{3}{*}{$\begin{array}{l}2009 \\
\text { Proj. }\end{array}$} \\
\hline & & & Q1 & Q2 & Q3 & Q4 & Year & \\
\hline & & & \multicolumn{5}{|c|}{ Prog. } & \\
\hline Overall revenues and grants & 23.6 & 21.6 & 5.3 & 5.3 & 5.8 & 6.0 & 22.4 & 22.6 \\
\hline Total revenues & 18.9 & 20.5 & 5.2 & 4.9 & 5.3 & 5.4 & 20.7 & 21.6 \\
\hline Tax revenues & 16.5 & 17.8 & 4.8 & 4.5 & 4.8 & 4.3 & 18.5 & 19.1 \\
\hline Income and profit tax & 2.1 & 2.4 & 0.7 & 0.6 & 0.6 & 0.5 & 2.4 & 2.5 \\
\hline Payroll taxes & 2.0 & 2.0 & 0.4 & 0.5 & 0.6 & 0.5 & 2.0 & 2.0 \\
\hline Property taxes & 0.8 & 0.7 & 0.2 & 0.2 & 0.2 & 0.2 & 0.8 & 0.8 \\
\hline Taxes on goods and services & 9.7 & 10.9 & 3.1 & 2.8 & 3.0 & 2.6 & 11.5 & 12.1 \\
\hline VAT & 7.3 & 8.3 & 2.3 & 2.2 & 2.3 & 2.1 & 8.9 & 9.2 \\
\hline Excises and other internal indirect taxes & 2.5 & 2.6 & 0.8 & 0.7 & 0.7 & 0.6 & 2.7 & 2.8 \\
\hline International trade and operations tax & 1.8 & 1.8 & 0.4 & 0.4 & 0.4 & 0.5 & 1.7 & 1.8 \\
\hline Non-tax revenues & 2.4 & 2.7 & 0.3 & 0.4 & 0.5 & 1.1 & 2.3 & 2.5 \\
\hline Grants $1 /$ & 4.7 & 1.1 & 0.1 & 0.4 & 0.5 & 0.6 & 1.7 & 1.0 \\
\hline Of which: PIP financing & 0.6 & 0.8 & 0.1 & 0.4 & 0.5 & 0.1 & 1.2 & 0.6 \\
\hline Total expenditures and lending minus repayments & 21.9 & 27.8 & 6.7 & 7.7 & 8.1 & 7.8 & 30.4 & 29.1 \\
\hline Current expenditures & 15.0 & 13.6 & 2.5 & 4.0 & 4.0 & 4.8 & 15.4 & 16.9 \\
\hline Expenditures on goods and services & 10.4 & 9.4 & 1.7 & 3.2 & 3.0 & 3.1 & 10.9 & 12.3 \\
\hline Wages and salaries & 4.1 & 4.1 & 0.8 & 1.0 & 1.4 & 1.4 & 4.6 & 5.3 \\
\hline Others & 6.3 & 5.3 & 0.8 & 2.2 & 1.6 & 1.8 & 6.3 & 7.0 \\
\hline Interest payments & 0.5 & 0.5 & 0.1 & 0.1 & 0.1 & 0.2 & 0.6 & 0.7 \\
\hline Transfers and subsidies & 4.1 & 3.8 & 0.8 & 0.8 & 1.0 & 1.4 & 3.9 & 3.9 \\
\hline Capital expenditures & 6.8 & 14.1 & 3.6 & 3.7 & 3.8 & 2.9 & 14.0 & 11.3 \\
\hline Externally financed PIP & 3.1 & 8.6 & 2.9 & 2.5 & 2.3 & 1.5 & 9.2 & 7.1 \\
\hline Of which: loans from China & 0.0 & 5.8 & 2.4 & 1.3 & 1.3 & 0.2 & 5.2 & 3.3 \\
\hline Domestically financed & 3.7 & 5.5 & 0.7 & 1.2 & 1.5 & 1.4 & 4.8 & 4.2 \\
\hline Lending minus repayments $2 /$ & 0.1 & 0.1 & 0.6 & 0.0 & 0.2 & 0.0 & 0.9 & 0.9 \\
\hline Overall balance (incl. externally financed PIP) & 1.7 & -6.2 & -1.4 & -2.4 & -2.3 & -1.8 & -8.0 & -6.4 \\
\hline Overall balance (excl. ext. financed PIP, PIP related grants) 3/ & 0.8 & 1.6 & 1.3 & -0.4 & -0.5 & -0.3 & 0.0 & 0.0 \\
\hline Overall balance (excl. externally financed PIP and all grants) $3 /$ & 0.1 & 1.3 & 1.3 & -0.4 & -0.5 & -0.8 & -0.4 & -0.4 \\
\hline Total financing (incl. externally financed PIP) & -1.7 & 6.2 & 1.4 & 2.4 & 2.3 & 1.8 & 8.0 & 6.4 \\
\hline Net external & 1.6 & 6.9 & 2.5 & 1.8 & 1.6 & 1.3 & 7.2 & 6.4 \\
\hline Disbursements & 2.9 & 7.8 & 2.7 & 2.1 & 1.8 & 1.5 & 8.1 & 6.9 \\
\hline Program loans & 0.4 & 0.0 & 0.0 & 0.0 & 0.0 & 0.1 & 0.1 & 0.4 \\
\hline Project loans & 2.5 & 7.8 & 2.7 & 2.1 & 1.8 & 1.4 & 8.0 & 6.5 \\
\hline Amortization & -1.3 & -0.8 & -0.2 & -0.2 & -0.2 & -0.3 & -0.9 & -0.5 \\
\hline Net domestic & -3.3 & -0.7 & -1.1 & 0.6 & 0.8 & 0.5 & 0.8 & 0.0 \\
\hline NBT 4/ & -4.1 & -1.1 & -0.9 & 0.5 & 0.7 & 0.3 & 0.5 & -0.1 \\
\hline Commercial banks & 0.0 & -0.4 & -0.2 & 0.1 & 0.1 & 0.1 & 0.1 & 0.1 \\
\hline Gross proceeds from privatization & 0.6 & 0.1 & 0.0 & 0.0 & 0.0 & 0.0 & 0.2 & 0.1 \\
\hline Other nonbank and discrepancy & 0.2 & 0.6 & 0.1 & 0.0 & 0.0 & 0.0 & 0.0 & 0.0 \\
\hline \multicolumn{9}{|l|}{ Memorandum items: } \\
\hline Nominal GDP (in millions of somoni) & 9,272 & 12,780 & & & & & 16,135 & 19,337 \\
\hline
\end{tabular}

Sources: Tajik authorities; and Fund staff estimates.

1/ 2006 grants figure includes the MDRI debt relief of 3.5 percent of GDP.

2/ Includes SM140 mn lending to the cotton sector in 2008.

3/ 2006 overall balance excludes the MDRI debt relief that is reflected in grants. The balance also differs from the officially

reported outcome due to a difference in recording the conversion of an EU loan into a grant.

4/ The historical financing figures in this table are used on estimated flows, not differences between end-period stocks. 
Table 3. Tajikistan: General Government Operations, 2006-09 (In millions of somoni; unless otherwise indicated)

\begin{tabular}{|c|c|c|c|c|c|c|c|c|}
\hline & \multirow{3}{*}{$\begin{array}{l}2006 \\
\text { Act. }\end{array}$} & \multirow{3}{*}{$\begin{array}{r}2007 \\
\text { Est. }\end{array}$} & \multicolumn{5}{|c|}{2008} & \multirow{3}{*}{2009} \\
\hline & & & \multirow[t]{2}{*}{ Q1 } & \multirow[t]{2}{*}{ Q2 } & \multirow{2}{*}{$\frac{\text { Q3 }}{\text { Prog. }}$} & \multirow[t]{2}{*}{ Q4 } & \multirow[t]{2}{*}{ Year } & \\
\hline & & & & & & & & \\
\hline Overall revenues and grants & 2,190 & 2,763 & 855 & 851 & 936 & 970 & 3,611 & 4,375 \\
\hline Total revenues & 1,754 & 2,621 & 832 & 784 & 853 & 875 & 3,343 & 4,182 \\
\hline Tax revenues & 1,533 & 2,274 & 778 & 724 & 775 & 702 & 2,979 & 3,702 \\
\hline Income and profit tax & 194 & 305 & 107 & 96 & 101 & 86 & 390 & 476 \\
\hline Payroll taxes & 189 & 253 & 72 & 74 & 89 & 84 & 319 & 382 \\
\hline Property taxes & 77 & 92 & 27 & 34 & 34 & 36 & 131 & 157 \\
\hline Taxes on goods and services & 902 & 1,398 & 501 & 455 & 483 & 423 & 1,861 & 2,332 \\
\hline VAT & 675 & 1,061 & 379 & 350 & 371 & 333 & 1,432 & 1,784 \\
\hline Excises and other internal indirect taxes & 227 & 337 & 122 & 105 & 111 & 90 & 428 & 548 \\
\hline International trade and operations tax & 171 & 226 & 71 & 65 & 68 & 73 & 278 & 356 \\
\hline Sales taxes & 87 & 95 & 21 & 22 & 17 & 43 & 102 & 145 \\
\hline Import duties & 84 & 131 & 50 & 44 & 51 & 30 & 176 & 210 \\
\hline Non-tax revenues & 221 & 347 & 53 & 60 & 77 & 174 & 364 & 480 \\
\hline Grants 1/ & 436 & 142 & 24 & 67 & 83 & 94 & 268 & 192 \\
\hline Of which: PIP financing & 59 & 107 & 24 & 67 & 83 & 20 & 194 & \\
\hline Total expenditures and lending minus repayments & 2,032 & 3,554 & 1,089 & 1,245 & 1,312 & 1,252 & 4,898 & 5,622 \\
\hline Current expenditures & 1,393 & 1,740 & 410 & 649 & 652 & 777 & 2,488 & 3,266 \\
\hline Expenditures on goods and services & 967 & 1,197 & 268 & 509 & 476 & 508 & 1,761 & 2,372 \\
\hline Wages and salaries & 384 & 525 & 131 & 161 & 225 & 225 & 742 & 1,019 \\
\hline Others & 583 & 672 & 137 & 348 & 252 & 283 & 1,019 & 1,353 \\
\hline Interest payments & 46 & 59 & 16 & 16 & 21 & 39 & 91 & 133 \\
\hline Transfers and subsidies & 380 & 484 & 126 & 124 & 154 & 231 & 635 & 761 \\
\hline Subsidies and other current transfers & 34 & 39 & 15 & 12 & 17 & 34 & 79 & 95 \\
\hline Capital expenditures & 634 & 1,806 & 576 & 594 & 620 & 475 & 2,264 & 2,180 \\
\hline Externally financed PIP & 291 & 1,100 & 464 & 402 & 373 & 249 & 1,488 & 1,368 \\
\hline Of which: loans from China & 0 & 746 & 392 & 210 & 210 & 29 & 841 & \\
\hline Domestically financed & 342 & 707 & 112 & 191 & 247 & 226 & 777 & 812 \\
\hline Lending minus repayments $2 /$ & 6 & 8 & 103 & 2 & 40 & 1 & 146 & 175 \\
\hline Overall balance (incl. externally financed PIP) & 158 & -791 & -234 & -394 & -376 & -283 & $-1,287$ & $-1,247$ \\
\hline Overall balance (excl. externally financed PIP and PIP related grants) & 70 & 202 & 206 & -59 & -86 & -54 & 8 & 3 \\
\hline Overall balance (excl. externally financed PIP and all grants) 3/ & 13 & 167 & 206 & -59 & -86 & -128 & -67 & -71 \\
\hline Total financing (incl. externally financed PIP) & -158 & 791 & 234 & 394 & 376 & 283 & 1,287 & 1,247 \\
\hline Net external & 145 & 884 & 406 & 297 & 254 & 205 & 1,163 & 1,238 \\
\hline Disbursements & 269 & 992 & 440 & 335 & 290 & 248 & 1,313 & 1,328 \\
\hline Program loans & 37 & 0 & 0 & 0 & 0 & 19 & 19 & 78 \\
\hline Project loans & 232 & 992 & 440 & 335 & 290 & 229 & 1,294 & 1,250 \\
\hline Amortization & -124 & -108 & -34 & -38 & -36 & -42 & -150 & -90 \\
\hline Net domestic & -303 & -94 & -173 & 97 & 123 & 78 & 124 & 9 \\
\hline NBT 4/ & -379 & -136 & -147 & 76 & 107 & 51 & 86 & -29 \\
\hline Commercial banks & -3 & -47 & -39 & 15 & 15 & 22 & 13 & 14 \\
\hline Gross proceeds from privatization & 57 & 17 & 5 & 6 & 6 & 8 & 25 & 24 \\
\hline Other nonbank and discrepancy & 22 & 73 & 8 & 0 & -5 & -3 & 0 & 0 \\
\hline
\end{tabular}

Sources: Tajik authorities; and Fund staff estimates.

1/ 2006 grants figure includes the MDRI debt relief of 3.5 percent of GDP.

2/ Includes SM140 mn lending to the cotton sector in 2008.

3/ 2006 overall balance excludes the MDRI debt relief that is reflected in grants. The balance also differs from the officially

reported outcome due to a difference in recording the conversion of an EU loan into a grant.

4/ The historical financing figures in this table are used on estimated flows, not differences between end-period stocks. 
Table 4. Tajikistan: Accounts of the National Bank of Tajikistan, 2005-08

(End-of-period stock; unless otherwise specified) 1/

\begin{tabular}{|c|c|c|c|c|c|c|c|}
\hline & & \multirow{2}{*}{$\begin{array}{r}\frac{2006}{\text { Dec. }} \\
\text { Act. }\end{array}$} & \multirow{2}{*}{$\frac{2007}{\text { Dec }}$} & \multicolumn{4}{|c|}{2008} \\
\hline & $\frac{\text { Dec. }}{\text { Act. }}$ & & & March & June & Sept & $\mathrm{Dec}$ \\
\hline \multicolumn{8}{|c|}{ (In millions of U.S. dollars; unless otherwise specified) } \\
\hline Net foreign assets $1 /$ & 103 & 217 & 271 & 298 & 301 & 322 & 357 \\
\hline Gross assets & 251 & 275 & 360 & 384 & 387 & 400 & 410 \\
\hline Gross liabilities & 147 & 59 & 88 & 86 & 86 & 78 & 54 \\
\hline Net international reserves & -30 & 87 & 66 & 93 & 96 & 117 & 151 \\
\hline Gross reserves & 117 & 136 & 118 & 143 & 146 & 159 & 169 \\
\hline Gross short-term liabilities & 147 & 49 & 53 & 50 & 50 & 42 & 18 \\
\hline Official exchange rate (Sm/US\$) & 3.199 & 3.427 & 3.465 & 3.453 & $\ldots$ & $\ldots$ & $\ldots$ \\
\hline Accounting exchange rate (Sm/US\$) & 3.435 & 3.435 & 3.435 & 3.435 & 3.435 & 3.435 & 3.435 \\
\hline \multicolumn{8}{|c|}{ (In millions of somoni) } \\
\hline Net foreign assets $1 /$ & 355 & 744 & 932 & 1,024 & 1,035 & 1,107 & 1,225 \\
\hline Gross assets & 862 & 946 & 1,236 & 1,319 & 1,330 & 1,374 & 1,409 \\
\hline Gross liabilities & 507 & 202 & 304 & 295 & 295 & 267 & 184 \\
\hline Net international reserves & 355 & 778 & 1,054 & 1,147 & 1,157 & 1,229 & 1,347 \\
\hline Gross reserves & 862 & 946 & 1,236 & 1,319 & 1,330 & 1,374 & 1,409 \\
\hline Gross short-term liabilities & 507 & 167 & 182 & 173 & 173 & 145 & 62 \\
\hline Net domestic assets & 333 & 277 & 500 & 420 & 454 & 528 & 551 \\
\hline Net credit to general government & -232 & -636 & -772 & -919 & -844 & -737 & -687 \\
\hline Credit to the private sector $2 /$ & 596 & 937 & 1,172 & 1,188 & 1,188 & 1,187 & 1,186 \\
\hline Other items, net & -31 & -23 & 100 & 152 & 110 & 79 & 52 \\
\hline NBT bills & -27 & -4 & -10 & 0 & 0 & -31 & -58 \\
\hline Reserve money & 687 & 1,021 & 1,432 & 1,445 & 1,489 & 1,635 & 1,776 \\
\hline Currency in circulation & 581 & 883 & 1,123 & 1,136 & 1,195 & 1,307 & 1,415 \\
\hline Bank reserves & 103 & 134 & 248 & 277 & 262 & 296 & 329 \\
\hline \multicolumn{8}{|l|}{ Of which: } \\
\hline Required reserves & 66 & 66 & 139 & 187 & 196 & 219 & 236 \\
\hline Other bank deposits & 37 & 68 & 109 & 90 & 65 & 78 & 93 \\
\hline \multirow[t]{2}{*}{ Other deposits } & 4 & 4 & 61 & 32 & 32 & 32 & 32 \\
\hline & \multicolumn{7}{|c|}{ (12-month change as a percent of initial reserve money stock) } \\
\hline Net international reserves & 12.2 & 46.8 & -5.7 & 7.8 & 12.4 & 11.1 & 16.4 \\
\hline Net domestic assets & 16.6 & -8.1 & 21.8 & 15.1 & 18.4 & 5.0 & 3.6 \\
\hline Reserve money & 28.8 & 48.5 & 40.2 & 42.1 & 41.8 & 16.2 & 24.1 \\
\hline Memorandum items: & & & & & & & \\
\hline Pledged international reserves (in millions of US\$) & 133 & 140 & 241 & 241 & 241 & 241 & 241 \\
\hline Credits to the cotton sector (in millions of somoni) & 378 & 571 & 885 & 893 & 893 & 893 & 893 \\
\hline
\end{tabular}

Sources: National Bank of Tajikistan; and Fund staff estimates.

1/ Based on accounting exchange rates: SM3.435=US $\$ 1$, SDR $1=$ US $\$ 1.65$, and gold price of 978.3 per troy ounce (end-March 2008 rates).

2/ Credits to private sector includes credits to the cotton sector. 
Table 5. Tajikistan: Monetary Survey, 2005-08

(In millions of somoni, end-of-period stock; unless otherwise specified) 1/

\begin{tabular}{|c|c|c|c|c|c|c|c|}
\hline & \multirow{3}{*}{$\frac{2005}{\frac{\text { Dec. }}{\text { Act. }}}$} & \multirow{3}{*}{$\frac{2006}{\frac{\text { Dec. }}{\text { Act. }}}$} & \multirow{3}{*}{$\frac{2007}{\frac{\text { Dec. }}{\text { Est. }}}$} & \multicolumn{4}{|c|}{2008} \\
\hline & & & & \multirow{2}{*}{$\begin{array}{l}\text { March } \\
\text { Est. }\end{array}$} & \multirow{2}{*}{\multicolumn{2}{|c|}{$\begin{array}{l}\text { June } \quad \text { Sept. } \\
\text { Prog. } 1 /\end{array}$}} & \multirow[t]{2}{*}{ Dec. } \\
\hline & & & & & & & \\
\hline Net foreign assets & -200 & 58 & 83 & 157 & 146 & 198 & 295 \\
\hline National Bank of Tajikistan & 355 & 744 & 932 & 1024 & 1035 & 1107 & 1225 \\
\hline Commercial banks & -555 & -686 & -849 & -868 & -888 & -909 & -930 \\
\hline Net domestic assets & 1,168 & 1,486 & 2,672 & 2,626 & 2,710 & 2,977 & 3,131 \\
\hline Net credit to general government & -267 & -674 & -857 & $-1,044$ & -953 & -831 & -759 \\
\hline Credit to the private sector & 1,671 & 2,403 & 3,794 & 3,928 & 3,968 & 4,118 & 4,204 \\
\hline Other items, net & -235 & -243 & -265 & -257 & -304 & -309 & -314 \\
\hline Broad money & 958 & 1,535 & 2,736 & 2,771 & 2,844 & 3,163 & 3,413 \\
\hline Somoni broad money & 674 & 1,046 & 1,447 & 1,411 & 1,383 & 1,538 & 1,660 \\
\hline Currency outside banks & 534 & 825 & 1,037 & 999 & 949 & 1,055 & 1,138 \\
\hline Deposits & 140 & 220 & 410 & 412 & 435 & 483 & 521 \\
\hline Foreign currency deposits & 284 & 489 & 1,289 & 1,359 & 1,461 & 1,625 & 1,753 \\
\hline Bills payable $2 /$ & 10 & 8 & 19 & 12 & 12 & 12 & 12 \\
\hline \multicolumn{8}{|l|}{ Memorandum items: } \\
\hline Actual/accounting exchange rate (Sm/US\$, eop) & 3.44 & 3.44 & 3.44 & 3.44 & 3.44 & 3.44 & 3.44 \\
\hline Broad money (12-month percent change) & 29.9 & 60.2 & 78.2 & 73.4 & 68.1 & 50.4 & 24.7 \\
\hline Credit to the private sector (12-month percent change) & 29.8 & 43.8 & 57.9 & 49.8 & 35.8 & 22.9 & 10.8 \\
\hline Year-to-year average of quarterly velocity $3 /$ & 2.1 & 1.8 & 1.6 & 1.5 & 1.4 & 1.3 & 1.3 \\
\hline Money multiplier $4 /$ & 1.4 & 1.5 & 1.9 & 1.9 & 1.9 & 1.9 & 1.9 \\
\hline
\end{tabular}

Sources: National Bank of Tajikistan; and Fund staff estimates.

1/ Based on accounting exchange rates: SM3.435=US $\$ 1$, SDR $1=$ US $\$ 1.65$, and gold price of 978.3 per troy ounce (end-March 2008 rates).

2/ Liabilities to cotton financiers related to domestic cotton financing.

3/ Quarterly GDP divided by end-quarter broad money; four-quarter average.

4/ Broad money divided by reserve money. 
Table 6. Tajikistan: Balance of Payments, 2005-2008 (In millions of US dollars)

\begin{tabular}{|c|c|c|c|c|c|c|c|c|}
\hline & \multirow{3}{*}{$\begin{array}{r}2005 \\
\text { Act. }\end{array}$} & \multirow{3}{*}{2006} & \multirow{3}{*}{$\begin{array}{r}2007 \\
\text { Est. }\end{array}$} & \multicolumn{5}{|c|}{2008} \\
\hline & & & & Q1 & Q2 & Q3 & Q4 & Year \\
\hline & & & & \multicolumn{5}{|c|}{ Prog. } \\
\hline Current account & -62 & -79 & -414 & -266 & -126 & 116 & -178 & -429 \\
\hline Balance on goods and services $1 /$ & -620 & -961 & $-1,788$ & -509 & -510 & -516 & -825 & $-2,333$ \\
\hline Balance on goods & -622 & -986 & $-1,673$ & -500 & -502 & -452 & -759 & $-2,187$ \\
\hline Exports & 346 & 350 & 385 & 77 & 94 & 93 & 120 & 402 \\
\hline Imports & -968 & $-1,336$ & $-2,058$ & -577 & -595 & -545 & -879 & $-2,589$ \\
\hline Of which: projects financed by China & 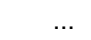 & $\ldots$ & -163 & -42 & -42 & -42 & -42 & -168 \\
\hline Balance on services & 2 & 25 & -115 & -9 & -8 & -63 & -65 & -146 \\
\hline Of which: aluminium tolling fee & 116 & 173 & 183 & 60 & 60 & 60 & 60 & 239 \\
\hline Of which: projects financed by China & & & -54 & 42 & 42 & 42 & 42 & -56 \\
\hline Balance on income & -79 & -101 & -111 & -26 & -29 & -29 & -35 & -120 \\
\hline Balance on transfers & 636 & 984 & 1,484 & 268 & 413 & 661 & 682 & 2,024 \\
\hline Of which: migrants' remittances, net & 526 & 903 & 1,416 & 277 & 446 & 722 & 728 & 1,957 \\
\hline Capital and financial account & 73 & 183 & 409 & 303 & 129 & -95 & 210 & 520 \\
\hline Capital transfers & 19 & 127 & 31 & 6 & 18 & 22 & 12 & 52 \\
\hline Of which: MDRI debt relief & $\ldots$ & 99 & $\ldots$ & $\ldots$ & $\ldots$ & $\ldots$ & $\ldots$ & $\ldots$ \\
\hline Public sector (net) & 48 & 35 & 253 & 108 & 78 & 67 & 48 & 301 \\
\hline Disbursements & 86 & 82 & 308 & 117 & 89 & 77 & 66 & 350 \\
\hline Of which: projects financed by China & $\ldots$ & $\ldots$ & 217 & & & & & 224 \\
\hline Amortization & -38 & -37 & -35 & -9 & -11 & -10 & -13 & -44 \\
\hline FDI & 55 & 66 & 160 & 45 & 45 & 45 & 45 & 180 \\
\hline Commercial bank NFA (- increase) & 191 & 27 & 44 & -15 & 5 & 5 & 5 & 1 \\
\hline Electricity credit & 6 & 18 & 6 & $\ldots$ & $\ldots$ & $\ldots$ & $\ldots$ & $\ldots$ \\
\hline NBT pledged deposits & -14 & -6 & -102 & $\ldots$ & $\ldots$ & $\ldots$ & $\ldots$ & $\ldots$ \\
\hline Other short-term capital and errors and omissions $2 /$ & -58 & -92 & -4 & 158 & -18 & -235 & 95 & -19 \\
\hline Overall balance & 11 & 104 & -5 & 36 & 3 & 20 & 32 & 92 \\
\hline Use of international reserves (- increase) & -27 & -20 & 5 & -36 & -3 & -13 & -10 & -62 \\
\hline Financing items & 16 & -84 & $\ldots$ & $\ldots$ & $\ldots$ & -7 & -22 & -29 \\
\hline IMF (net) & 15 & -85 & $\ldots$ & $\ldots$ & $\ldots$ & -7 & -22 & -29 \\
\hline Purchases/disbursements & 29 & 14 & $\ldots$ & $\ldots$ & $\ldots$ & $\ldots$ & $\ldots$ & $\ldots$ \\
\hline Repurchases/repayments & 14 & 99 & $\ldots$ & $\ldots$ & $\ldots$ & 7 & 22 & 29 \\
\hline Financing gap & $\ldots$ & $\ldots$ & $\ldots$ & $\ldots$ & $\ldots$ & $\ldots$ & $\ldots$ & $\ldots$ \\
\hline In percent of GDP & $\cdots$ & $\ldots$ & $\cdots$ & & & & & $\ldots$ \\
\hline \multicolumn{9}{|l|}{ Memorandum items: } \\
\hline GDP (in millions of U.S. dollars) & 2,311 & 2,811 & 3,712 & $\ldots$ & $\ldots$ & $\ldots$ & $\ldots$ & 4,300 \\
\hline Current account balance in percent of GDP & -2.7 & -2.8 & -11.2 & $\ldots$ & $\ldots$ & $\ldots$ & $\ldots$ & -10.0 \\
\hline Current account balance excluding projects financed by China & -2.7 & -2.8 & -5.3 & $\ldots$ & $\ldots$ & $\ldots$ & $\ldots$ & -4.8 \\
\hline Gross reserves & 91 & 111 & 107 & 143 & 146 & 159 & 169 & 169 \\
\hline (in months of imports) 3/ & 0.9 & 0.7 & 0.5 & & & & & 0.8 \\
\hline Debt service on PPG external debt 4/ & 60 & 49 & 49 & $\ldots$ & $\ldots$ & $\ldots$ & $\ldots$ & 111 \\
\hline (in percent of exports of goods) & 17.5 & 14.0 & 12.8 & $\ldots$ & $\ldots$ & $\ldots$ & $\ldots$ & 27.7 \\
\hline Total PPG external sector debt & 897 & 910 & 1231 & $\ldots$ & $\ldots$ & $\ldots$ & $\ldots$ & 1492 \\
\hline (in percent of GDP) & 38.8 & 32.4 & 33.2 & $\ldots$ & $\ldots$ & $\ldots$ & $\ldots$ & 34.7 \\
\hline
\end{tabular}

Sources: Tajik authorities; and Fund staff estimates.

1/ Starting from 2005, the export and import figures reflect the transition to tolling arrangement for aluminum exports.

2/ Includes accumulation of foreign currency balances by residents of Tajikistan.

3/ Excluding electricity, which is on barter basis, and imports related to projects financed by China.

4/ Data for 2006 exclude $\$ 99$ million IMF repurchase under MDRI assistance. 
Table 7. Tajikistan: Medium-Term Balance of Payments, 2005-10

(In millions of US dollars)

\begin{tabular}{|c|c|c|c|c|c|c|}
\hline & 2005 & 2006 & 2007 & 2008 & 2009 & 2010 \\
\hline & Act. & Act. & Est. & Prog. & \multicolumn{2}{|c|}{ Proj. } \\
\hline Current account & -62 & -79 & -414 & -429 & -401 & -238 \\
\hline Balance on goods and services $1 /$ & -620 & -961 & $-1,788$ & $-2,333$ & $-2,461$ & $-2,447$ \\
\hline Balance on goods & -622 & -986 & $-1,673$ & $-2,187$ & $-2,315$ & $-2,347$ \\
\hline Exports & 346 & 350 & 385 & 402 & 411 & 436 \\
\hline Imports & -968 & $-1,336$ & $-2,058$ & $-2,589$ & $-2,726$ & $-2,783$ \\
\hline Of which: projects financed by China & $\ldots$ & $\ldots$ & -163 & -168 & -122 & $\ldots$ \\
\hline Balance on services & 2 & 25 & -115 & -146 & -145 & -101 \\
\hline Of which: aluminium tolling fee & 116 & 173 & 183 & 239 & 251 & 276 \\
\hline Of which: projects financed by China & $\ldots$ & $\ldots$ & -54 & -56 & -41 & $\ldots$ \\
\hline Balance on income & -79 & -101 & -111 & -120 & -124 & -124 \\
\hline Balance on transfers & 636 & 984 & 1,484 & 2,024 & 2,183 & 2,333 \\
\hline Of which: public grants & 4 & 17 & 10 & 20 & 19 & 15 \\
\hline Of which: migrants' remittances, net & 526 & 903 & 1,416 & 1,957 & 2,126 & 2,287 \\
\hline Capital and financial account & 73 & 183 & 409 & 520 & 476 & 298 \\
\hline Capital transfers & 19 & 127 & 31 & 52 & 30 & 25 \\
\hline Of which: MDRI debt relief & $\ldots$ & 99 & $\ldots$ & $\ldots$ & $\ldots$ & $\ldots$ \\
\hline Public sector (net) & 48 & 45 & 273 & 306 & 306 & 146 \\
\hline Disbursements & 86 & 82 & 308 & 350 & 340 & 179 \\
\hline Of which: projects financed by China & $\ldots$ & $\ldots$ & 217 & 224 & 163 & $\ldots$ \\
\hline AsDB program loans & $\ldots$ & $\ldots$ & $\ldots$ & 5 & 20 & 20 \\
\hline Amortization & -38 & -37 & -35 & -44 & -34 & -33 \\
\hline FDI & 55 & 66 & 160 & 180 & 180 & 180 \\
\hline Commercial bank NFA (- increase) & 191 & 27 & 44 & 1 & -8 & -7 \\
\hline Electricity credit & 6 & 18 & 6 & $\ldots$ & $\ldots$ & $\ldots$ \\
\hline NBT pledged deposits & -14 & -6 & -102 & $\ldots$ & $\ldots$ & $\ldots$ \\
\hline Other short-term capital and errors and omissions $2 /$ & -58 & -92 & -4 & -19 & -33 & -45 \\
\hline Overall balance & 11 & 104 & -5 & 92 & 75 & 60 \\
\hline Use of international reserves (- increase) & -27 & -20 & 5 & -62 & -60 & -60 \\
\hline Financing items & 16 & -84 & $\ldots$ & -29 & -15 & $\ldots$ \\
\hline IMF (net) & 15 & -85 & $\ldots$ & -29 & -15 & $\ldots$ \\
\hline Purchases/disbursements & 29 & 14 & $\ldots$ & $\ldots$ & $\ldots$ & $\ldots$ \\
\hline Repurchases/repayments & 14 & 99 & $\ldots$ & 29 & 15 & $\ldots$ \\
\hline Financing gap & $\cdots$ & $\ldots$ & $\ldots$ & $\ldots$ & $\ldots$ & ... \\
\hline In percent of GDP & $\ldots$ & $\ldots$ & $\ldots$ & $\ldots$ & $\ldots$ & $\ldots$ \\
\hline Memorandum items: & & & & & & \\
\hline GDP (in millions of U.S. dollars) & 2,311 & 2,811 & 3,712 & 4,300 & 4,955 & 5,455 \\
\hline Current account balance in percent of GDP & -2.7 & -2.8 & -11.2 & -10.0 & -8.1 & -4.4 \\
\hline Current account balance excluding projects financed by China & -2.7 & -2.8 & -5.3 & -4.8 & -4.8 & -4.4 \\
\hline Terms of trade (percent change) & 5.1 & 31.2 & 4.1 & -8.1 & -9.2 & -10.7 \\
\hline Gross reserves & 91 & 111 & 107 & 169 & 229 & 289 \\
\hline (in months of imports) 3/ & 0.9 & 0.7 & 0.5 & 0.8 & 1.0 & 1.2 \\
\hline Debt service on PPG external debt 4/ & 60 & 49 & 49 & 111 & 82 & 59 \\
\hline (in percent of exports of goods) & 17.5 & 14.0 & 12.8 & 27.7 & 20.0 & 13.6 \\
\hline Total PPG external sector debt & 897 & 910 & 1,231 & 1,492 & 1,779 & 1,914 \\
\hline (in percent of GDP) & 38.8 & 32.4 & 33.2 & 34.7 & 35.9 & 35.1 \\
\hline
\end{tabular}

Sources: Tajik authorities; and Fund staff estimates.

1/ Starting from 2005, the export and import figures reflect the transition to tolling arrangement for aluminum exports.

$2 /$ Includes accumulation of foreign currency balances by residents of Tajikistan.

3/ Excluding electricity, which is on barter basis, and imports related to projects financed by China.

4/ Data for 2006 exclude $\$ 99.2 \mathrm{mln}$. IMF repurchase under MDRI assistance. 
Table 8. Tajikistan: Medium-Term Macroeconomic Projections, 2005-12

\begin{tabular}{|c|c|c|c|c|c|c|c|c|}
\hline & & & & 2008 & 2009 & 2010 & 2011 & 2012 \\
\hline & 2005 & 2006 & 2007 & \multicolumn{5}{|c|}{ Staff Projections } \\
\hline \multicolumn{9}{|l|}{ National income and prices } \\
\hline Nominal GDP (in millions of somoni) & 7,201 & 9,272 & 12,780 & 16,135 & 19,337 & 22,138 & 25,109 & 28,479 \\
\hline Nominal GDP (in millions of U.S. dollars) & 2,311 & 2,811 & 3,712 & 4,300 & 4,955 & 5,455 & 6,007 & 6,614 \\
\hline Real GDP (annual percent change) & 6.7 & 7.0 & 7.8 & 5.0 & 7.0 & 7.0 & 7.0 & 7.0 \\
\hline GDP per capita (in U.S. dollars) & 357 & 430 & 561 & 643 & 733 & 798 & 869 & 957 \\
\hline GDP deflator (annual percent change) & 9.6 & 20.3 & 27.9 & 20.2 & 12.0 & 7.0 & 6.0 & 6.0 \\
\hline Consumer prices (12-month percent change, e.o.p.) & 7.1 & 12.5 & 19.8 & 15.0 & 9.0 & 6.0 & 6.0 & 6.0 \\
\hline \multicolumn{9}{|l|}{ General government finances } \\
\hline $\begin{array}{l}\text { Total revenue and grants } \\
\text { Of which: }\end{array}$ & 20.1 & 23.6 & 21.6 & 22.4 & 22.6 & 23.5 & 23.4 & 23.4 \\
\hline Tax revenue & 16.6 & 16.5 & 17.8 & 18.5 & 19.1 & 20.0 & 20.0 & 20.1 \\
\hline $\begin{array}{l}\text { Total expenditure } \\
\text { Of which: }\end{array}$ & 23.0 & 21.9 & 27.8 & 30.4 & 29.1 & 26.7 & 27.7 & 27.7 \\
\hline Current expenditure & 15.1 & 15.0 & 13.6 & 15.4 & 16.9 & 17.9 & 18.0 & 18.0 \\
\hline Externally-financed public investment program (PIP) & 3.4 & 3.1 & 8.6 & 9.2 & 7.1 & 3.4 & 4.4 & 4.4 \\
\hline Fiscal balance (excluding the PIP) $1 /$ & 0.5 & 0.8 & 1.6 & 0.0 & 0.0 & -0.3 & -0.3 & -0.2 \\
\hline \multicolumn{9}{|l|}{ Savings/investment balances } \\
\hline External current account balance & -2.7 & -2.8 & -11.2 & -10.0 & -8.1 & -4.4 & -5.6 & -5.5 \\
\hline Overall fiscal balance (including the PIP) 1 / & -2.9 & -1.8 & -6.2 & -8.0 & -6.4 & -3.2 & -4.3 & -4.2 \\
\hline Net savings (S-I) of the private sector $2 /$ & 0.2 & -1.0 & -5.0 & -2.0 & -1.7 & -1.1 & -1.3 & -1.2 \\
\hline \multicolumn{9}{|l|}{ External sector $3 /$} \\
\hline Exports of goods and services & 601 & 656 & 767 & 856 & 896 & 970 & 1,048 & 1,113 \\
\hline Imports of goods and services & 1,221 & 1,618 & 2,555 & 3,188 & 3,357 & 3,417 & 3,705 & 3,916 \\
\hline Current account balance & -62 & -79 & -414 & -429 & -401 & -238 & -339 & -362 \\
\hline Gross international reserves & 91 & 111 & 107 & 169 & 229 & 289 & 319 & 359 \\
\hline Gross international reserves, excluding projects financed by China (in months of imports) & 0.9 & 0.7 & 0.5 & 0.8 & 1.0 & 1.2 & 1.2 & 1.3 \\
\hline Total public and publicly guaranteed external debt (in percent of GDP) & 38.8 & 32.4 & 33.2 & 34.7 & 35.9 & 35.1 & 34.5 & 33.9 \\
\hline
\end{tabular}

Sources: Tajik authorities; and Fund staff estimates.

1/ 2006 overall balance excludes the MDRI debt relief that is reflected in grants.

2/ Defined as the external current account balance less the overall fiscal balance (including the PIP).

3/ Starting from 2005, export and import figures reflect the transition to tolling arrangement for aluminum exports.

Therefore, they show a sudden drop compared to earlier years. 


\section{AtTAChMent I}

10 June, 2008

His Excellency

Mr. Dominique Strauss-Kahn

Managing Director

International Monetary Fund

$70019^{\text {th }}$ Street, N.W.

Washington, D.C. 20431

Dear Mr. Strauss-Kahn:

I would like to take this opportunity to express my gratitude to the International Monetary Fund for its support of Tajikistan at this critical moment for our economy. We regret the recent misreporting incident and assure you that we are determined and ready to address the institutional weaknesses that led to it.

I am pleased to inform you that during the April 17-30, 2008 mission, we reached understandings with Fund staff on a Staff Monitored Program (SMP) covering the period June 1-December 31, 2008. The SMP establishes a macroeconomic framework that will support our efforts at economic stabilization and implementation of structural reforms to help address the current problems faced by Tajikistan. At the core of this program are measures to strengthen the autonomy and governance of the National Bank of Tajikistan (NBT), aiming to address weaknesses exposed by the recent incident of misreporting. In this connection, after agreeing with Fund staff on the appropriate terms of reference, we have launched the hiring process of a reputable independent international auditing firm to conduct a special audit of the NBT.

The Government believes that the policies outlined in the Memorandum of Economic and Financial Policies (MEFP) will enhance the prospects for achieving the objectives of our economic program for 2008. We intend to remain in close consultation with Fund staff and provide timely information necessary for monitoring economic developments and implementation of policies under the SMP.

In addition, the Government stands ready to take any further measures that might be required to ensure that the overall objectives of the program are attained. In order to enhance transparency of our economic policies, we find it appropriate that all the documents related to the SMP be published on the Fund's website. In addition, we will also publish all key findings of the special audit of the NBT.

Once the SMP is successfully completed, we hope to begin discussions on a new program that could be supported by the Fund under the Poverty Reduction and Growth Facility.

Your Excellency, I would like to resume my best regards to you,

/s/

Emomali Rakhmon

President of the Republic of Tajikistan 


\section{Attachment II. Memorandum of Economic and Financial Policies for the REPUBLIC OF TAJIKISTAN}

\section{INTRODUCTION}

1. This memorandum outlines the economic and financial policies of the Government of Tajikistan for the remainder of 2008, which are aimed at restoring macroeconomic stability and reestablishing the credibility of our policy reform efforts in light of the recent misreporting episode. We are hereby asking Fund staff to monitor the implementation of these policies, with a view to building a policy track record that could support an eventual request for a new Poverty Reduction and Growth Facility (PRGF) arrangement.

\section{THE FOCUS OF THE PROGRAM}

2. The main objectives of the proposed Staff-Monitored Program (SMP) will be twofold:

- Addressing potential shortcomings in the National Bank of Tajikistan's (NBT) governance and legal framework. Efforts in this area will be closely linked to the conduct of a special audit of the NBT and other involved parties. The audit will look specifically into the circumstances surrounding the misreporting of data to the Fund by the NBT and the origin and extent of related financial operations and transactions. Moreover, as recommended by the FSAP team, a number of changes will be introduced to the NBT and other banking laws to address the governance problems exposed by the misreporting episode, including conflict of interest.

- $\quad$ Providing an appropriate macroeconomic policy framework for price stability and growth. The current low level of international reserves and the limits on the flexibility of monetary policy imposed by the insolvency of the NBT, call for the continuation of a prudent fiscal policy stance and the implementation of well-defined debt strategy aimed at limiting the risks of future debt distress.

3. In addition, these efforts will be complemented by the implementation of measures consistent with the broad outlines of a comprehensive strategy that tackles the fundamental problems of the cotton sector. We are in the process of identifying and developing the measures in consultation with the World Bank and the Asian Development Bank (ADB).

\section{A. The Macroeconomic Framework for 2008}

4. The $\mathbf{2 0 0 8}$ macroeconomic framework envisages a slowdown in real GDP growth to about 5 percent and targets a limited reduction in inflation to 15 percent. While we expect a strong rebound in economic activity in the second half of 2008 , to be supported by the continuing stimulus from remittances and the commissioning of the Sangtuda 1 hydropower plant, the weather-related problems experienced in the first quarter of the year are likely to put a dent in the growth rate for the year as a whole. Specifically, the agricultural 
sector is likely to underperform as it was particularly hit hard by the frost. While inflation is targeted to slow from its current rate of about 20 percent, it will remain in double digits largely on account of the impact on the CPI of high international food and fuel prices and required adjustments in electricity and gas tariffs. The current account deficit is expected to remain relatively high at about 10 percent of GDP, but should remain fully financed by FDI flows and official concessional project finance disbursements. Gross official reserves are targeted to increase, but import coverage will remain relatively low at less than one month.

\section{B. The Special Audit of the NBT and Related Legal Reforms}

\section{We have agreed with Fund staff on the terms of reference (ToR) for a special} audit of the NBT (prior action). In order to launch the hiring process of a reputable independent international auditing firm (prior action), on May 14, 2008, we forwarded these ToR to potential candidate firms and requested bids. As progress is made in the conduct of the audit, we will be sharing interim reports with Fund staff. The main objectives of the special audit will be:

- To assess the effectiveness of the governance structure and control environment at the NBT, including ascertaining the nature of the governance breakdown and weaknesses that led to the credits provided to Kredit Invest being excluded from the NBT's audited balance sheets and the nondisclosure of significant pledges and guarantees.

- $\quad$ To review the transactions involving balances linked to information that was not provided to the Fund and the previous external auditor of the NBT. This review would also seek to confirm the legitimacy of the payments to third parties by Kredit Invest.

- To verify the accuracy of data reported to the Fund after the discovery of the misreporting episode and review existing procedures for reporting data, with the final objective of making recommendations for strengthening controls to ensure the accuracy and veracity of the data.

\section{The program also includes measures to strengthen the autonomy and} governance of the NBT. Specifically, by end-December 2008, we will amend the NBT law, and other related legislation as needed (structural benchmark), to address shortcomings in the areas of:

- $\quad$ Terms of appointment and removal of board members. The NBT Chairman and his/her deputies will be appointed for fixed terms, which will not coincide with the electoral cycle. Moreover, the reasons for their dismissal will be clearly defined in the law.

- $\quad$ Conflict of interest. We will introduce clearer provisions for what constitutes conflict of interest behavior and introduce sanctions to be applied in the case of violations. 
- $\quad$ Disclosure of beneficial ownership. We will introduce requirements for the identification and disclosure of beneficial ownership of companies registered with the Agency for Securities in the Ministry of Finance and other authorized entities, including financial institutions.

7. We have requested technical assistance from the World Bank to draft these legal amendments, with the aim of conducting a more comprehensive assessment of the financial sector legal framework to bring it up to international standards.

\section{Monetary and Exchange Rate Policies}

8. Our monetary program envisages an expansion in reserve money in line with nominal GDP growth. Nevertheless, given that changes in velocity are still difficult to predict in the current environment, should signs emerge that underlying inflation is not falling as planned, the NBT will tighten monetary conditions in consultation with Fund staff, while aiming to maintain its international reserves accumulation program target. In addition,

- $\quad$ the NBT will refrain from issuing credits to the private sector, except to commercial banks (including Amonatbank) for liquidity and lender of last resort purposes; and

- tightly enforce reserve requirements on all deposit liabilities of all financial institutions without exception.

9. Moreover, in order to support our inflation objective and help the country cope with external shocks, the NBT remains committed to a managed floating exchange rate regime, with intervention in the foreign exchange market limited to smoothing operations and to ensuring that targeted reserves gains are achieved.

\section{Fiscal Policy and Public Financial Management}

10. The fiscal program for 2008 envisages a balanced budget (excluding the Public Investment Program [PIP]). Despite the slowdown in economic activity early in the year, the strong revenue performance of 2007 carried over to the first four months of 2008. Much

of this strong revenue performance was due to continuing strength in remittances inflows, but also reflected comprehensive efforts to improve tax administration. Given the fiscal overperformance in 2007 (a surplus in excess of 1 1 $\frac{1}{2}$ percent of GDP), aiming for a balanced budget will still allow for an increase in real expenditures, including for tackling the basic needs of those hit hard by the recent weather-related events and providing financing to the cotton sector transparently through the budget. The PIP will be limited to about 9 percent of GDP in 2008.

11. While public financial management has improved, we still need to shore up the monitoring and corporate governance of state-owned enterprises. In this connection, we intend to: 
- $\quad$ Issue tender papers for an audit of Talco's and Barki Tajik's 2007 financial operations by an internationally recognized auditing company by end-September 2008 (structural benchmark);

- $\quad$ Issue a government decree by end-September 2008 establishing a unit in the Ministry of Finance for regular monitoring of financial operations of the 10 SOEs with the highest annual turnover (structural benchmark). The decree will require the SOEs to provide the Ministry of Finance and Ministry of Economic Development and Trade draft annual financial plans for review and approval before the beginning of each financial year and submit their quarterly financial statements and their external audit reports.

\section{E. Debt Management Strategy}

12. In order to mitigate increased risks of debt problems, we will adopt a well-defined debt strategy. The strategy includes:

- $\quad$ No new contracting or guaranteeing of nonconcessional debt.

- A commitment that any new concessional foreign debt disbursement over the next three years will be consistent with the government's borrowing plan already approved by Parliament in January 2008.

\section{This strategy should serve to stabilize the external public and publicly} guaranteed debt-to-GDP ratio in the medium term at about 40 percent. Moreover, in order to supplement these efforts, we will continue to strengthen our debt data management framework, including by establishing a contingent liability reporting regime by end-December 2008 to cover state-owned enterprises, public institutions and the NBT (structural benchmark).

\section{F. Cotton Sector Reforms}

14. We are taking steps to reinvigorate our cotton sector reform efforts. As a stopgap measure, we have put in place a transparent cotton financing mechanism that uses budgeted public funds for lending to farmers through commercial banks. To safeguard the financial position of the banks involved in this scheme, we will be introducing appropriate performance-based management contracts for banks to make efforts to collect the outstanding sums without unduly penalizing them for delinquencies. We are also, in consultation with the World Bank and the ADB, in the process of designing a comprehensive strategy to deal more permanently with the long-standing problems of the sector. The strategy will deal with the two key aspects of this problem: a) improving the profitability of the sector; and b) resolving the outstanding stock of debt. On the former, we are currently taking steps to implement Decree 111, which addresses distortions in cotton production, including issues of freedom to farm and ability to use land rights as collateral. As regards the outstanding debt stock, we are currently in discussions with Kredit Invest's external creditors seeking to improve the terms of the obligations that were guaranteed by the NBT, including 
through the extension of maturities and a reduction of interest rates. As part of the process of resolving Kredit Invest, we are also exploring the possibility of swapping some of its external obligations for assets that were used by domestic borrowers (cotton investors) to collateralize their own obligations to Kredit Invest. In this connection, we will endeavor to maximize loan recoveries from these domestic borrowers. In support of our cotton sector reforms, by end-September 2008 we will also issue a decree that will abolish the practice of the NBT issuing export licenses beginning with the 2008 crop.

\section{G. Program Monitoring}

15. We have reached understandings with Fund staff on a set of quantitative financial targets and structural benchmarks for the remainder 2008 (Tables 1 and 2), which are defined in the attached Technical Memorandum of Understanding (TMU) and will be used to monitor progress in implementing the program. Specifically, the TMU defines the quantitative targets, the program adjustors, and specifies reporting requirements. The program comprises indicative quantitative targets and structural benchmarks for September and December 2008. There will be two reviews of the program, one in October 2008 and the other in January 2009. The first review will focus mainly on assessing progress in the conduct of the special audit of the NBT. 


\begin{tabular}{|c|c|}
\hline Areas of reform & Implementation Date \\
\hline $\begin{array}{l}\text { 1. Reach understandings with Fund staff on the Terms of Reference for the special audit of the NBT } \\
\text { and other involved parties, as defined in the terms of reference. } \\
\text { 2. Request bids for the selection of an internationally reputable audit firm } \\
\text { to conduct the special audit of the NBT and other involved parities defined in the terms of reference. } \\
\text { 3. Provide interim reports, as noted in the terms of reference of the special audit of the NBT } \\
\text { to Fund staff. } \\
\text { 4. NBT will refrain from issuing new credits to the private sector, except to commercial banks } \\
\text { (including Amonatbank) for liquidity and lender of last resort purposes. These involve credits that } \\
\text { are issued in the absence of a competitive auction or on non-market terms and conditions. } \\
\text { 5. Make legislative changes to: (i) appoint the NBT chairman and his deputies for fixed terms } \\
\text { that do not coincide with the electoral cycle, and outline the reasons for their dismissal explicitly in the } \\
\text { law; (ii) require official identification and disclosure of beneficial owners of companies registered } \\
\text { with the Agency for Securities in the Ministry of Finance and other authorized entities, } \\
\text { including financial institutions; (iii) clarify the provisions covering conflicts of interest for the NBT } \\
\text { management and staff and require them to disclose their personal holdings in financial institutions } \\
\text { and excuse themselves from official decisions affecting those institutions. } \\
\text { 6. Issue tender papers for an audit of Talco's and Barki Tajik's } 2007 \text { financial operations } \\
\text { by an internationally recognized auditing company. } \\
\text { monitoring of financial operations of the } 10 \text { SOE's with the highest annual turnover and require these } \\
\text { enterprises to provide the MoF and Ministry of Economic Development and Trade draft annual } \\
\text { financial plans for review and approval before the beginning of each financial year and submit } \\
\text { their quarterly financial statements and their external audit reports. } \\
\text { to cover state-owned enterprises, public institutions and the NBT. } \\
\text { tsue a government decree to establish a supervision unit in the Ministry of Finance (MoF) for regular }\end{array}$ & $\begin{array}{l}\text { End-Sept. } 2008 \\
\text { End-Dec. } 2008 \\
\text { End-Sept. } 2008\end{array}$ \\
\hline
\end{tabular}


Table 2. Tajikistan: Quantitative Indicators under the 2008 Staff Monitored Program

(In millions of somoni, unless otherwise indicated)

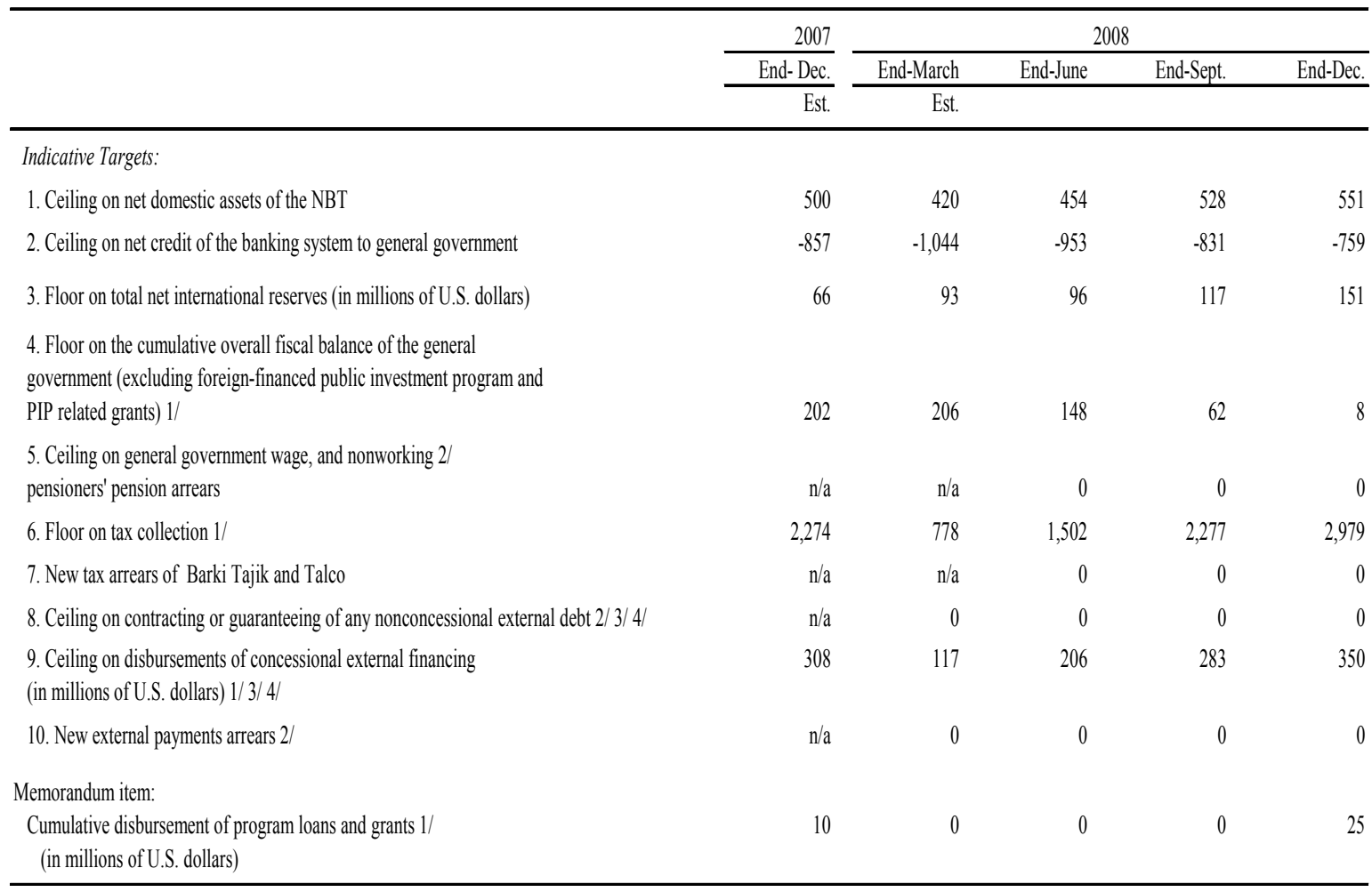

Sources: Tajik authorities; and Fund staff estimates.

1/ Cumulative from January 1 of the year.

2/ Continuous quantitative targets.

3/ Short, medium and long-term debt by the government, NBT or any other agency acting on behalf of the government

as defined in the Technical Memorandum of Understanding, excluding normal trade-related loans.

4/ Excluding the obligations resulting from the restructuring of the cotton debt. 


\section{AtTaChment III. TeChnical Memorandum OF Understanding FOR THE REPUBLIC OF TAJIKISTAN}

16. Performance under the Staff-Monitored Program will be assessed based on observance of quantitative targets and structural benchmarks. This annex defines the variables that constitute quantitative targets under the program, and sets out the reporting requirements and frequency of data provision by the government and the NBT.

17. For the purpose of program monitoring, U.S. dollar-denominated components of the balance sheet will be valued at the program exchange rate, and other foreign currency denominated items will be valued at cross rates between the program exchange rate of the U.S. dollar and current official exchange rates of the U.S. dollar against those currencies. The program exchange rate for the U.S. dollar is SM 3.435 = \$1 (the end-March 2008). The SDR components will be valued at the program SDR exchange rate (SDR $1=\$ 1.647)$. Official gold holdings shall be valued at $\$ 978.25$ per troy ounce.

\section{Quantitative TARgets}

\section{A. Fiscal Balance of the General Government}

Table 1. Ceiling on the Cumulative Overall Balance of the General Government

\begin{tabular}{lc}
\hline & (In millions of somoni) \\
\hline Cumulative balance from January 1, 2008 to: & \\
March 31, 2008 & 206 \\
June 30, 2008 & 148 \\
September 30, 2008 & 62 \\
December 31, 2008 & 8
\end{tabular}

\section{Definitions}

18. The general government budget is defined to include the republican budget, local (including municipal) budgets, and all extra-budgetary funds at all levels of general government, including the Agency on Social Protection and Pensions, but excluding the externally financed public investment program. The overall cash balance of the general government is defined from the financing side as the sum of the following: 
(i) The change in stocks of the NBT's net claims on the general government, which include all deposits of the general government with the NBT, counterpart deposits (which reflect balance of payment and/or general budget support from the IFIs and other donors), NBT loans and advances to the general government, NBT holdings of government securities, bank restructuring costs, and the privatization account (where proceeds from the privatization of state property are held);

(ii) The change in net claims (transactions) on the general government of the rest of the domestic banking system which are defined to include the net position of the general government with respect to other domestic commercial bank assets (loans, overdrafts, cash advances, holdings of treasury bills or other securities) and liabilities (deposits, etc.);

(iii) The change in net claims (transactions) on the general government of domestic nonbank institutions and households is defined to include net sales of Treasury bills, bonds or other government securities to nonbank institutions and households (including nonresidents and nonresident financial institutions), plus any other increase in liabilities of the general government to domestic nonbank institutions or households;

(iv) Gross proceeds from the privatization of state property are defined as all receipts originating from the sale of state property; and

(v) Net foreign financing of the general government which is defined as the difference between gross disbursements of foreign financing excluding the externallyfinanced public investment program and amortization of government debt to foreign financial and nonfinancial institutions, plus principal arrears and debt rescheduling.

\section{B. Total Tax Collections under the Budget}

Table 2. Floor on Total Tax Collections Under the Budget

\begin{tabular}{lc}
\hline & (In millions of somoni) \\
\hline Cumulative balance from January 1, 2008 to: & \\
March 31, 2008 & 778 \\
June 30, 2008 & 1,502 \\
September 30, 2008 & 2,227 \\
December 31, 2008 & 2,979 \\
\hline
\end{tabular}




\section{Definitions}

19. Tax collections include all taxes collected under the budget. Regarding internal taxation, the definition excludes any proceeds from loans, or other banking system credits, the issuance of securities, or from the sale of state assets. Custom revenues are defined to include customs duties and other taxes (including VAT) on international trade and transactions.

\section{Limits on the Stock of Net Domestic Assets of the NBT}

Table 3. Ceiling on the Stock of Net Domestic Assets of the NBT

\begin{tabular}{lc}
\hline & (In millions of somoni) \\
\hline March 31, 2008 & 420 \\
June 30, 2008 & 454 \\
September 30, 2008 & 528 \\
December 31, 2008 & 551 \\
\hline
\end{tabular}

\section{Definitions}

20. Net domestic assets (NDA) of the NBT are defined as: reserve money minus net foreign assets of the NBT. Reserve money is composed of currency in circulation, required reserves, other bank reserves, and deposits of nongovernment nonbanks with the NBT. The value of the net foreign assets of the NBT is calculated as the difference between NBT's claims on nonresidents and its liabilities to nonresidents. The NBT's net domestic assets comprise the following assets and liabilities: net credit to the general government, claims on banks, credit to the economy, and other items net (OIN). OIN includes the foreign exchange revaluation and capital accounts of the NBT.

\section{Adjustors}

21. The ceiling on net domestic assets of the NBT will be adjusted upward by the value in domestic currency of any existing pledged deposits of the NBT that is not renewed by the deposit holder. The pledged deposits amounted to \$241 million as of end-March 2008.

\section{Limits on Net Credit of the Banking System to General Government}

Table 4. Ceiling on Net Credit of the Banking System to General Government 1/

\begin{tabular}{lc}
\hline & (In millions of somoni) \\
\hline March 31, 2008 & $-1,044$ \\
June 30, 2008 & -953 \\
September 30, 2008 & -831 \\
December 31, 2008 & -759 \\
\hline
\end{tabular}

1/ Calculated at fixed program exchange rate. 


\section{E. Net International Reserves}

Table 5. Floor under the Stock of Net International Reserves of the NBT

(In millions of U.S. dollars)

March 31, 2008

June 30, 2008

September 30, 2008

December 31, 2008
93

96

117

151

\section{Definitions}

22. Total net international reserves of the NBT are defined as the difference between total gross international reserves of the NBT and total reserve liabilities of the NBT. Total gross international reserves of the NBT are defined to include NBT's holdings of monetary gold, SDRs, convertible currencies in cash or in nonresident banks that are readily available and any reserve position in the IMF. Also included are holdings of foreign currencydenominated securities issued by governments or central banks of OECD member states. Excluded are capital subscriptions in foreign financial institutions, nonliquid assets of the NBT, convertible currency denominated claims on domestic banks and other residents, assets in nonconvertible currencies, foreign assets pledged as collateral or otherwise encumbered and the net forward position, if any (defined as the difference between the face value of foreign currency denominated NBT off balance sheet claims on nonresidents and foreign currency obligations to both residents and nonresidents). Reserve liabilities of the NBT are defined as the sum of outstanding IMF credit and liabilities of the NBT to nonresidents with an original maturity of up to and including one year.

23. The Fund staff will be informed of details of any gold sales, purchases, pledges, guarantees or swap operations during the program period, and any resulting changes in the level of gross foreign reserves that arise from revaluation of gold will be excluded from gross reserves (as defined above).

\section{Adjustors}

24. The floor on the net international reserves of the NBT will be adjusted upward for any conversion of existing pledged deposits into long-term debt. 


\section{F. Limits on Short-, Medium-, and Long-Term External Debt}

Table 6. Cumulative Ceiling on the Contracting

or Guaranteeing of Non-Concessional External Debt

\begin{tabular}{lcc}
\hline & $0-1$ Year Maturity & Over 1 Year Maturity \\
\hline During the period from end-December 2007 to: & & \\
March 31, 2008 & 0 & 0 \\
June 30, 2008 & 0 & 0 \\
September 30, 2008 & 0 & 0 \\
December 31, 2008 & 0 & 0 \\
& & \\
\hline
\end{tabular}

\section{Definitions}

25. The external debt limits (short-, medium-, and long term) apply to the government of Tajikistan, the National Bank of Tajikistan and any other agency acting on behalf of the government, including but not limited to state-owned banks. For short-, medium- and longterm external debt, the target applies not only to debt as defined in point No. 9 of the Guidelines on Performance Criteria with Respect to Foreign Debt (Decision No. 12274(00/85), adopted August 24, 2000), but also to commitments contracted or guaranteed and for which value has not been received.

26. The definition of debt set forth in point No. 9 of the guidelines reads as follows: "(a) For the purposes of this guideline, the term "debt" will be understood to mean a current, i.e., not contingent, liability, created under a contractual arrangement through the provision of value in the form of assets (including currency) or services, and which requires the obligor to make one or more payments in the form of assets (including currency) or services, at some future points in time; these payments will discharge the principal and/or interest liabilities under the contract. Debts can take a number of forms, the primary ones being as follows: (a) loans, i.e., advances of money to obligor by the lender made on the basis of an undertaking that the obligor will repay the funds in the future (including deposits, bonds, debentures, commercial loans, and buyers' credits) and temporary exchanges of assets that are equivalent to fully collateralized loans under which the obligor is required to repay the funds and usually pay interest, by repurchasing the collateral from the buyer in the future (such as repurchase agreements and official swap arrangements); (b) suppliers' credits, i.e., contracts where the supplier permits the obligor to defer payments until some time after the date on which the goods are delivered or services are provided; and (c) leases, i.e., arrangements under which property is provided which the lessee has the right to use one or more specified period(s) of time that are usually shorter than the total expected service life of the property, while the lessor retains the title to the property. For the purpose of the guideline, the debt is the present value (at the inception of the lease) of all lease payments expected to be made during the period of the agreement excluding those payments that cover the operation, repair or maintenance of the property. (b) Under the definition of debt set out in point 9(a) above, arrears, penalties, and judicially awarded damages arising from the 
failure to make payment under a contractual obligation that constitutes debt are debt. Failure to make payment on an obligation that is not considered debt under this definition (e.g., payment on delivery) will not give rise to debt."

27. External debt limits apply to the contracting or guaranteeing of new non-concessional short term external debt (with an original maturity of up to and including one year), and to the contracting or guaranteeing of new nonconcessional medium- and long-term external debt (with original maturities of more than one year).

28. Excluded from the external debt limits are loans contracted for the purpose of debt rescheduling or refinancing if the terms of the new loan are more favorable. IMF credit is excluded from the external debt limits. The rollover of the existing guarantees will be excluded from the debt limits. If pledged reserves of the NBT were to be securitized, these amounts will also be excluded from the debt limits. The indicative target on new nonconcessional short-term external debt will not apply to loans classified as international reserve liabilities of the NBT (liabilities of the NBT to nonresidents with an original maturity of up to and including one year). Normal import-related financing is excluded from the target on new short-term external debt.

29. Debt falling within the external debt limits that are denominated in currencies other than the U.S. dollar shall be valued in U.S. dollars at the exchange rate prevailing at the time the contracting or guaranteeing takes place or at the exchange rate stipulated in the contract.

30. For the purposes of the program, the guarantee of a debt arises from any explicit legal obligation of the government or the NBT or any other agency acting on behalf of the government to service such a debt in the event of nonpayment by the debtor (involving payments in cash or in kind), or indirectly through any other obligation of the government or the NBT or any other agency acting on behalf of the government to finance a shortfall incurred by the debtors.

31. Concessional debt is defined as debt with a grant element equivalent of 35 percent or more. The grant element is calculated using the commercial interest reference rates (CIRRs) plus a margin, which will be taken as those for February 15, 2008 until this is revised in the program. For debts of an original maturity of 15 years or more, the average CIRR over the last 10 years will be used, while the average of CIRRs of the preceding six-month period will be used to assess the concessionality of debts with original maturities of less than 15 years. To the ten-year and six month averages of CIRRs, the following margins will be added: 0.75 percent for repayment periods of less than 15 years; 1 percent for 15-19 years; 1.15 percent for 20-29 years; and 1.25 percent for 30 and more years. In general, grant element is calculated as value of the loan minus present discounted value of future payments over the loan value (multiplied by 100). Present discounted value is sum of all future discounted payments (both amortization and interest), where the discount rate corresponds to the commercial interest reference rate mentioned above. 


\section{Continuous Quantitative Targets}

\section{A. No Directed Credits by the NBT}

32. The NBT will not issue any directed credits except to banks for liquidity and lender of last resort purposes. These involve credits that are issued in the absence of a competitive auction or on non-market terms and conditions. This requirement will be monitored on the basis of changes in the NBT's balance sheets supported by the NBT's regular reporting on credit related activities.

\section{No New External Payments Arrears}

33. No new external payments arrears shall be accumulated at any time under the program, excluding those which are subject to negotiation among creditors. External payments arrears are defined as debt service overdue for 30 days or more arising in respect of obligations incurred directly, guaranteed, or converted into interstate debt by the government of Tajikistan or the NBT, including penalties or interest charges.

\section{Ceiling on General Government Wage and Nonworking Pensioners Pension Arrears}

34. For purposes of the target, arrears on general government and nonworking pensioners' pension shall be monitored as any shortfall in monthly disbursements of wages and pensions. These payments are defined as overdue if they have come due at the end of the month and remain unpaid thereafter for 30 days or more. The program ceilings on the stock of wage and pension arrears are presented in Table 7 below:

Table 7. Stock of Wage and Pension Arrears

\begin{tabular}{lc}
\hline & (In millions of somoni) \\
\hline Cumulative from January 1, 2008 to: & \\
& \\
March 31, 2008 & 0 \\
June 30, 2008 & 0 \\
September 30, 2008 & 0 \\
December 31, 2008 & 0 \\
\hline
\end{tabular}

35. To permit monitoring as defined above, the government will provide data on actual wage payments (as part of the monthly budget execution statements submitted to the Fund staff). The Agency on Social Protection and Pensions will provide quarterly reports and statements of their operations. 


\section{E. Disbursements of Contracted Government and Government Guaranteed External Debt}

Table 8. Ceiling on Cumulative Disbursements of Contracted Government and Government Guaranteed External Debt

(In mln. of U.S. dollars)

\begin{tabular}{ll}
\hline Test dates: & \\
End-March 2008 & 117 \\
End-June 2008 & 206 \\
End-September 2008 & 283 \\
End-December 2008 & 350 \\
\hline & \\
\hline & In millions of U.S. dollars \\
2008 & 350 \\
2009 & 667 \\
2010 & 814 \\
\end{tabular}

\section{Definition}

The ceiling applies to disbursements of debt contracted or guaranteed by the government as defined in paragraphs 8-14 of this Annex. The ceiling on disbursements will be consistent with the government's borrowing plan already approved by Parliament in January 2008.

\section{Reporting ReQuirements Under the Program}

36. For monitoring program targets, the following data should be reported to the Fund.

Table 9. Data reporting frequency for program monitoring

\begin{tabular}{|c|c|c|}
\hline Data & Frequency & Lag \\
\hline 1. NBT analytical balance sheet & Weekly & 3 working days \\
\hline 2. NBT balance sheet & Monthly & 2 weeks \\
\hline 3. Commercial banks' and Kredit Invest balance sheets & Monthly & 4 weeks \\
\hline 4. Auction results for NBT certificates and treasury bills & Weekly & 1 week \\
\hline 5. Foreign exchange transactions & Weekly & 1 week \\
\hline $\begin{array}{l}\text { 6. Fiscal revenues, expenditures and financing } \\
\text { of budget execution, and extrabudgetary funds }\end{array}$ & Monthly & 4 weeks \\
\hline $\begin{array}{l}\text { 7. Reports and statements from the Agency on Social } \\
\text { Protection and Pensions }\end{array}$ & Quarterly & 6 weeks \\
\hline $\begin{array}{l}\text { 8. Arrears of budget entities and state owned } \\
\text { enterprises }\end{array}$ & Quarterly & 4 weeks \\
\hline $\begin{array}{l}\text { 9. Stocks, disbursements, guarantees, new contracts } \\
\text { of external debt including terms and disbursement profile. }\end{array}$ & Monthly & 4 weeks \\
\hline
\end{tabular}




\title{
INTERNATIONAL MONETARY FUND
}

\section{TAJIKISTAN}

\section{Staff-Monitored Program-Informational Annex}

\author{
June 10, 2008
}

Contents

Page

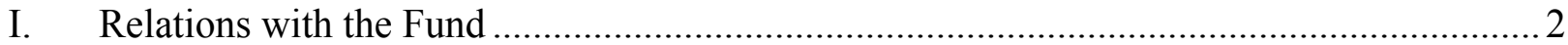

II. Relations with the World Bank Group.......................................................................

III. Relations with the Asian Development Bank ……….......................................................... 13

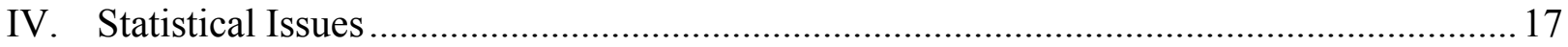




\section{ANNEX I. TAJIKISTAN: RELATIONS WITH THE FUND}

(As of April 30, 2008)

I. Membership Status: Joined April 27, 1993; Article VIII

II. General Resources Account:

Quota

$\begin{array}{rrr}\text { SDR million } & & \text { \% Quota } \\ 87.00 & & 100.00 \\ 87.00 & 100.00 \\ 0.00 & 0.00\end{array}$

III. SDR Department

Holdings

$\underline{\text { SDR million }}$

\% Allocation

2.37

N/A

IV. Outstanding Purchases and Loans

PRGF arrangements

\begin{tabular}{|c|}
\hline SDR million \\
\hline 29.40 \\
\hline
\end{tabular}

\section{Latest Financial Arrangements}

\begin{tabular}{llcccc} 
Type & \multicolumn{1}{c}{$\begin{array}{c}\text { Approval } \\
\text { Date }\end{array}$} & & $\begin{array}{c}\text { Expiration } \\
\text { Date }\end{array}$ & $\begin{array}{c}\text { Amount Approved } \\
\text { (SDR million) }\end{array}$ & $\begin{array}{c}\text { Amount Drawn } \\
\text { (SDR million) }\end{array}$ \\
\cline { 1 - 3 } PRGF & Dec 11, 2002 & Feb 10, 2006 & 65.00 & 65.00 \\
ESAF/PRGF & Jun 24, 1998 & Dec 24, 2001 & 100.30 & 78.28 \\
Stand-by & May 08, 1996 & Dec 07, 1996 & 15.00 & 15.00
\end{tabular}

VI. Projected Payments to Fund

(SDR million; based on existing use of resources and present holdings of SDRs):

\begin{tabular}{rrccc} 
& & Forthcoming & & \\
\hline 2008 & 2009 & 2010 & 2011 & 2012 \\
19.60 & 9.80 & & & \\
0.13 & 0.00 & & & \\
19.73 & 9.80 & & &
\end{tabular}




\section{Implementation of HIPC Initiative}

Not Applicable.

\section{Implementation of MDRI Assistance}

I. Total Debt Relief (SDR Million) 69.31

Of Which MDRI 69.31

HIPC --

II. Debt Relief by Facility (SDR Million)

\begin{tabular}{llll}
\multicolumn{4}{l}{ Eligible Debt } \\
\hline Delivery Date & $\underline{\text { GRA }}$ & $\underline{\text { PRGF }}$ & $\underline{\text { Total }}$ \\
January 2006 & N/A & 69.31 & 69.31
\end{tabular}

\section{Safeguards Assessment}

The previous safeguards assessment of the NBT was completed on July 23, 2003, which proposed specific measures to address a number of weaknesses, as reported in EBS/03/96. The next safeguards assessment is pending the results of the special audit of the NBT.

\section{Exchange Rate Arrangements}

The NBT maintains conventional peg exchange rate arrangement, as per 2008 reclassification of the regime from managed float. Since August 2005, the NBT has increased the frequency of calculating and announcing the official exchange rate from a weekly to a daily basis. The official exchange rate is based on all interbank transactions in foreign exchange.

With effect from December 9, 2004, the Republic of Tajikistan accepted the obligations of Article VIII, Sections 2, 3, and 4 of the Articles of Agreement. As a result, the Republic of Tajikistan maintains an exchange system that is free of restrictions on the making of payments and transfers for current international transactions, except for exchange restrictions maintained for security reasons that have been notified to the Fund pursuant to Executive Board decision No. 144-(52/51).

\section{FSAP Participation}

Tajikistan participated in the Financial Sector Assessment Program during 2007-8, and the FSSA document will be circulated to the Board shortly after the SMP document.

\section{Article IV Consultation}

The 2006 Article IV consultation was completed on March 28, 2007.

\section{Resident Representative}

Mr. Moers, Resident Representative of the Fund, started his assignment in Dushanbe in June 2006. 


\section{Technical Assistance}

The following list summarizes the technical assistance provided by the Fund to Tajikistan since 2002.

\section{Fiscal Affairs:}

July 2004

Revenue Administration Reform

December 2004

Poverty and Social Impact Analysis

June 2005

Public Financial Management

August 2005

Tax Policy and Administration

August 2006

Fiscal ROSC

May 2007

Public Financial Management (Budget Classification)

Monetary and Financial Systems:

July 2002

May 2006

\section{Statistics:}

October/November 2002

January 2003

February 2003

May 2003

April 2004

October 2004

June 2006

\section{Finance:}

May 2003

\section{Legal:}

March 2003

January 2004

May 2004
Multi-topic

Strengthening the Monetary Policy Framework and Liquidity Management

Money and Banking Statistics

Balance of Payments Statistics

Money and Banking Statistics

Balance of Payments Statistics

Data ROSC

General Data Dissemination System (GDDS)

Report on Monetary and Financial Statistics

Safeguards Assessment Follow-up

Tax Legislation

Tax Legislation

Tax Legislation 


\section{ANNEX II. TAJIKISTAN: RELATIONS WITH THE WORLD BANK}

(As of May 16, 2008)

Country Director: Ms. Annette Dixon

Country Economist: Mr. Sudharshan Canagarajah
Telephone: (202) 4737089

Telephone: (202) 4734458

\section{Partnership in Tajikistan's Development Strategy}

1. The Bank's Country Partnership Strategy, endorsed by the Bank's Board of Executive Directors on July 26, 2005, and updated on June 22, 2007, focuses on the following three priorities, which are in line with the country's Second Poverty Reduction Strategy (PRS 2) for 2007-2009:

- $\quad$ To improve business opportunities in rural and urban areas.

- $\quad$ To enhance and preserve the quality of the human capital.

- $\quad$ To improve energy service delivery and increase electricity exports.

2. In addition, in each of the above areas, the Bank Group and government agreed to work to improve government capacity and efficiency, and to reduce corruption by giving special emphasis to measures that increase transparency of resource use, reduce discretionary controls in business operations, and encourage the participation of users in the provision of services.

3. Macroeconomic management had aimed at establishing a stable economic environment through appropriate fiscal, monetary, exchange rate, and sustainable debt policies. However, macroeconomic management has recently worsened and the risk of debt distress has emerged. Tajikistan's long-term prospects remain positive only if the authorities move quickly to restore sound macroeconomic management and implement institutional reforms to improve the business climate, the financial sector, the agricultural sector, transparency and governance of large SOEs, and public financial management. Expansion in aluminum processing capacity to service continued high world demand, recovery in cotton production following institutional reforms, buoyant inflows of remittances, and the start of a large-scale Russian-financed hydroelectric power project and other infrastructure investments have the potential to support growth of 7-8 percent over the long term.

4. The IMF has taken the lead in assisting Tajikistan in enhancing macroeconomic stability. The Fund has encouraged the authorities to continue with fiscal consolidation, maintain a restrictive monetary policy, and conduct prudent debt management policy to enhance its sustainability.

5. The World Bank has taken the lead in the policy dialogue on structural issues, including poverty reduction measures, agriculture sector reforms, private sector development, institution building, budget planning, improving public service delivery, and governance. A range of instruments is used to conduct the dialogue. Policy based lending and technical 
assistance are supporting reforms in a number of sectors. The Programmatic Development Policy Grant (PDPG) - a three-year development policy financing program to support government's medium-term reform program — was approved in July 6, 2006; it is supporting the government in the areas of public administration reform, public expenditure management, social service delivery, and private sector development.

6. This broad-based approach is combined with sector projects in health, education, municipal infrastructure, power, agriculture, environment, and poverty alleviation. Over the last three years the Bank in collaboration with others has produced analytical work aimed at informing program design. A public expenditure and institutional review, a country procurement assessment report, a country financial accountability assessment, a public and civil service wage note and an update, and a remittances note have informed the public sector reform program. A trade diagnostic, an investment climate assessment, an aviation sector note, an energy utility reform review, the Central Asia Regional Electricity Export Potential Study, and an Agricultural Development Strategy have highlighted key reforms required to attract private sector investment. A poverty assessment update has deepened understanding of poverty and its dynamics, and is important for the design of policy reforms that effectively reduce poverty. Poverty and social impact assessments of cotton and energy reforms have helped promote dialogue with the government on issues of social protection. A programmatic public expenditure review is supporting Tajikistan's first public expenditure and financial accountability review, and analyzing the country's prospects for achieving the MDGs and long-term growth, and providing support for the introduction of the MTEF. Two public expenditure tracking surveys, one for health and one for education, will promote transparency and efficiency of expenditure in these sectors. A country environmental analysis has identified policy and capacity reforms to improve environmental management. 
Tajikistan: Collaboration of the World Bank and the IMF

\begin{tabular}{|c|c|c|c|}
\hline Area & $\begin{array}{l}\text { Specialized Advice from } \\
\text { Fund }\end{array}$ & $\begin{array}{l}\text { Specialized Advice } \\
\text { from Bank }\end{array}$ & Key Instruments \\
\hline $\begin{array}{l}\text { Economic } \\
\text { Framework/ } \\
\text { Management }\end{array}$ & $\begin{array}{l}\text { Monetary policy, exchange } \\
\text { rate, fiscal, and trade } \\
\text { policies, economic statistics }\end{array}$ & $\begin{array}{l}\text { Economic growth, } \\
\text { economic statistics }\end{array}$ & $\begin{array}{l}I M F \text { : Dialogue on performance } \\
\text { criteria and benchmarks on } \\
\text { monetary and fiscal targets. } \\
\text { Bank: PDPG and Strengthening } \\
\text { National Statistics System Project } \\
\text { under implementation. }\end{array}$ \\
\hline Budget & $\begin{array}{l}\text { Medium-term budget } \\
\text { framework, tax and customs } \\
\text { policy and administration, } \\
\text { and treasury and debt } \\
\text { management }\end{array}$ & $\begin{array}{l}\text { Budget management, } \\
\text { debt management and } \\
\text { statistics, public } \\
\text { expenditure and } \\
\text { financial management } \\
\text { reform }\end{array}$ & $\begin{array}{l}I M F \text { : Dialogue on performance } \\
\text { criteria on overall fiscal balance, } \\
\text { including PIP. Structural } \\
\text { benchmark on treasury } \\
\text { development. } \\
\text { Bank: PDPG, Public Sector } \\
\text { Reform Project (PSRP) under } \\
\text { implementation; TA for public } \\
\text { procurement (under } \\
\text { implementation) and external } \\
\text { audit (recently completed) with } \\
\text { grant funding from the } \\
\text { Institutional Development Fund } \\
\text { (IDF). Public Finance } \\
\text { Management Project (PFMP) } \\
\text { under preparation. }\end{array}$ \\
\hline $\begin{array}{l}\text { Public Sector } \\
\text { Reform }\end{array}$ & & $\begin{array}{l}\text { Civil service reform, } \\
\text { public sector wage } \\
\text { reform, public } \\
\text { administration reform, } \\
\text { health and education } \\
\text { financing }\end{array}$ & $\begin{array}{l}I M F \text { : Dialogue. } \\
\text { Bank: PDPG and PSRP, } \\
\text { Education Modernization Project } \\
\text { (EMP), Community and Basic } \\
\text { Health Project (CBHP) and } \\
\text { Education for All Fast Track } \\
\text { Initiative Catalytic Fund Grant } \\
\text { (FTI CF Grant) under } \\
\text { implementation. }\end{array}$ \\
\hline $\begin{array}{l}\text { Private and } \\
\text { Financial } \\
\text { Sector } \\
\text { Development }\end{array}$ & $\begin{array}{l}\text { Bank supervision; } \\
\text { development of the } \\
\text { interbank market and } \\
\text { increasing competition in } \\
\text { the banking sector. }\end{array}$ & $\begin{array}{l}\text { Pricing policies, } \\
\text { developing and } \\
\text { implementing the } \\
\text { privatization strategy, } \\
\text { farm privatization, } \\
\text { cotton debt resolution, } \\
\text { land reform. A grant } \\
\text { from FIRST for } \\
\text { financial sector reform } \\
\text { under preparation. }\end{array}$ & $\begin{array}{l}\text { IMF: Dialogue. } \\
\text { Bank: PDPG, Land Cadastre } \\
\text { Project and Cotton Sector } \\
\text { Modernization Project under } \\
\text { implementation. } \\
\text { TA for Private Sector } \\
\text { Development Strategy under } \\
\text { implementation with grant } \\
\text { funding from the IDF. }\end{array}$ \\
\hline
\end{tabular}


Other Sectors
Reforms in agriculture,

energy, health,

education, water and

sanitation, environment,

infrastructure.
Bank: PDPG, Education

Modernization, Community and

Basic Health, Municipal

Infrastructure Development,

Energy Loss Reduction, Dushanbe Water Supply, Land

Cadastre, Ferghana Valley Water,

Cotton Sector Modernization

Projects under implementation.

\section{World Bank Collaboration in Specific Areas}

\section{Areas in which the World Bank leads and there is little direct IMF involvement}

7. Areas in which the Bank leads and there is no direct IMF involvement are social sectors, infrastructure, and environment. In the social sphere, the Bank has carried out regular poverty assessments to monitor poverty and develop programs to alleviate poverty. It has prepared a poverty assessment update has been prepared on the basis of the 2003 living standards survey. Under the Strengthening National Statistics System Project, data collection of a new living standards survey was completed in 2007; analysis of the results is underway.

8. In education, the Bank is providing policy advice and investment resources. The ongoing Education Modernization Project (FY03) aims to upgrade the basic education system by supporting reforms in education financing and management, curriculum and textbooks development; teacher training and student assessment improvement, and upgrading school facilities. Tajikistan has also received grant funding from the Education for All Fast Track Initiative catalytic fund, administered by the Bank. Key issues in education, apart from infrastructure rehabilitation and capacity building, are pay reform, per capita financing and expenditure tracking. Assistance to this sector will continue through the PDPG, multi-donor education investment program, and the PSRP. The Bank is also helping with wage reform in education and a gradual move towards program-based budgeting in the sector through the PDPG. Jointly with the UNICEF the Bank assists the Government in the overall national education strategy formulation. The IMF has supported the education reform process, in close collaboration with the World Bank, through a PRGF structural benchmark requiring the drawing up of a fully costed education reform plan by the government.

9. In health, the Bank is helping the government to improve the effectiveness and efficiency of delivery - both of which are aimed at ensuring the poor access to a basic level of services. Through the Community and Basic Health Project and PDPG, the Bank is helping to strengthen the institutional capability (both at the center and district levels) to carry out health care reforms, to improve the system for budgeting and spending for basic health services, and pay reform. It is also supporting programs to prevent HIV/AIDS, malaria, and other communicable diseases from spreading. The Avian Fluenza Control and Human Pandemic Preparedness and Response is minimizing the threat of highly pathogenic avian influenza infection and other zoonoses posed to humans and poultry; and preparing for 
control and respond to influenza pandemics and other infectious disease emergencies in humans.

10. With regard to infrastructure development, the Bank is concentrating on upgrading basic utility services impacting the population at large and especially the poor. The Dushanbe Water Supply Project is helping the Dushanbe Municipality and the local water company to address the most critical deficiencies of water supply services. The Pamir Private Power Project is supporting improvements in reliability of electricity supply in the Gorno Badakshan region through private sector participation. The Energy Loss Reduction Project is helping to reduce commercial losses and improve the power and gas sector financial viability. The Municipal Infrastructure Project is providing support to improve urban infrastructure in big towns. The Bank has also been leading policy dialogue (under PDPG) on increasing efficiency/viability to ensure long-term growth prospects of the sector.

11. In agriculture, the Bank is concentrating on land reform, rehabilitation of irrigation and drainage infrastructure and introduction of market rules in procurement of inputs and marketing of outputs, as well as on the resolution of the cotton farm debts. The Land Registration and Cadastre Project is assisting the government with the land reform, issuance of land certificates and establishment of a modern cadastre system. The Ferghana Valley Project aims to increase water supply and efficiency of irrigation systems in the Ferghana Valley, and develop institutional capacity in land and water resources management. The Cotton Sector Recovery Project is helping provide assistance to improve the livelihoods of cotton farmers and to create conditions for sustainable growth of cotton production in selected, low-income areas. The project is doing this through assistance for cotton debt resolution, for an improved policy environment, and increased cotton output and profitability. Cross cutting policy issues for increasing productivity in cotton sectors are being addressed through the PDPG.

12. The Bank has supported programs to improve environmental management and to deal with natural disasters. A National Environment Action Plan was developed with the Bank's help. A Country Environmental Analysis has highlighted the country's most severe environmental issues and proposed measures to strengthen the legal and institutional framework for addressing them. The Emergency Flood Assistance and Lake Sarez Risk Mitigation Projects have helped the government to mitigate the consequences of frequent natural disasters and to build national capacity to address frequent natural hazards such as mudslides, rock-falls, avalanches, and seasonal floods.

\section{Areas in which the World Bank leads and its analysis serves as input into the IMF program}

13. The Bank leads the dialogue on structural reforms through the three-year PDPG program currently under implementation. Institution building and technical assistance in support of PDPG structural reforms come from several sources, including the PSRP, the Education Modernization Project, Fast Track Initiative Grant and the Community and Basic Health Project. 
14. The Bank and the Fund both support private sector development, including in the areas of financial transparency and improvements in the regulatory framework. The Bank has completed the Investment Climate Assessment and agriculture sector review to determine the most important impediments to private sector development. Tajikistan has participated in the Doing Business survey in 2006 and 2007 which provides insightful and cross-country comparison of the country's business environment. The Bank has supported the preparation of the government's private sector development strategy.

15. The Bank and the Fund support enterprise and farm privatization, including acceleration of privatization of medium and large enterprises, development of a plan to engage the private sector in Tajikistan's strategic enterprises and adoption of a strategy to privatize these enterprises in the medium-term, improvements to the corporate governance framework public enterprises, land reform, and restructuring of the cotton subsector. The Bank has completed the Investment Climate Assessment and agriculture sector review to identify obstacles to growth of agricultural productivity. With support of donors, the Bank has been providing technical support to the State Committee for Investments and State Property Management to implement long-term strategic privatization plan and promote private sector investment.

16. Regulatory reforms include (a) changing role of anti-monopoly agency and further strengthening its capacity, (b) improving inspection regime for businesses; and (c) streamlining licensing. In addition, under the PDPG, the Bank is assisting with separating in aviation the functions of policy-making, technical regulation, and accident investigation, with the aim of increasing the transparency and performance of operations. It is also helping with the restructuring the Tajik Air Company aimed at separating the airport, airline, and air traffic control.

17. While the Bank has taken the lead in privatization and in structural reforms in the private sector as described above, the IMF has also a strong interest in these areas since many of these reforms are critical to achieving macroeconomic stabilization and enhancing growth. Accordingly, there is a high degree of consultation and coordination between the two institutions on these matters.

\section{Areas of shared responsibility}

18. The Bank and the Fund are working jointly in the following four main areas (supported by the Bank's PDPG, grants, and several investment operations and the Fund's PRGF):

- Public sector management. The Bank has provided technical support to the government to develop a comprehensive public administration reform strategy. It includes policy actions in public administration reforms aimed at redefining the role of the state in line with the market economy needs, reorganizing key ministries, strengthening the internal control and audit function, and supporting the pay reform. The Bank is involved in civil service reform while the Fund is providing technical assistance in support of tax and customs administration and treasury management. 
- Budget planning and execution. Both institutions work on providing support for a treasury, adoption of new law on public finances introducing modern budgetary procedures and improved fiscal management, and switching from norm-based costing and allocation of expenditures to per capita based financing in education and health sectors. The Bank will continue to provide technical support, and mobilize donor funds, to implement the MTBF. The Bank will also continue to support public financial external and internal control reform through the PDPG, IDF grants, and the PSRP. The forthcoming Public Financial Management Project (FY09) will support treasury reform.

- Financial sector reforms. This area includes the acceleration of financial sector restructuring and closure of weak banks, a new regulatory framework for the establishment of nonbank intermediaries; significant changes in the tax code making tax authorities' access to bank accounts conditional on a court authorization. In terms of banking supervision, the IMF is monitoring the closure and merger of banks that do not satisfy prudential requirements. A Financial Sector Assessment Program (the country's first), carried out jointly by the Bank and the Fund, was discussed with the authorities in late 2007. The Bank has submitted an application to the FIRST trust fund to support implementation of the assessment's recommendations.

- Utilities reform. The Fund included in the PRGF benchmarks related to energy tariff rates, energy arrears, and collection rates with the aim to reduce quasi-fiscal activities and increase transparency. The Bank under the PDPG is supporting tariff reform aimed at reducing the quasi-fiscal deficit. It is also supporting the conducting of an audit of Barki Tajik's accounts, which is expected to be completed by August 2008. The Bank is providing financial assistance to help the government to repair the electricity and gas transmission system, to import the energy needed to meet periodic winter shortfalls, and to help implement the Energy Emergency Action Mitigation Plan. The Bank-supported Energy Loss Reduction Project is supporting implementation of reforms. The Bank is working closely together with the Asian Development Bank and the EBRD on utilities reform and restructuring of strategic enterprises such as the railways and airlines.

- Debt sustainability analysis: The Bank and the IMF have started carrying out joint DSAs. The first one was done in early 2006 and an update was completed in February 2007.

\section{Areas in which the IMF leads and its analysis serves as input into the World Bank program}

19. The Fund leads the dialogue on fiscal matters, setting the overall envelope for public expenditures. The Bank's work in key sectors, such as health, education and infrastructure, necessitates close cooperation. 
20. In the budgetary area, the Fund is taking the lead on tax reforms and budget preparation and execution. The IMF also leads the dialogue on polices to rationalize and contain expenditures in the public sector. These include policies regarding wage setting in both the public service, and defining the ceiling for public investment expenditures.

21. In these areas, the Bank takes into account the policy recommendations of the IMF and ensures that its own policy advice is consistent.

\section{Areas in which the IMF leads and there is no direct World Bank involvement}

22. The Fund takes the lead in the formulation and execution of fiscal and monetary policies, tax policy, external trade policies, and issues involving economic and financial statistics. However, the World Bank has recently provided an IDA project and catalyzed other donors' financing for the Strengthening National Statistics System Project.

\section{IDA Funding}

23. IDA funding to Tajikistan was provided in the form of 100 percent grant in FY06 and FY08; while in FY07 allocation was set with a 45 percent grant component. Tajikistan's eligibility for grants in outer years will be determined based on the annual Debt Sustainability Analyses. The indicative FY06-08 IDA funding is about SDR70 million; the FY06 commitment was SDR 30 million, FY07 commitment was around SDR 24 million, while the FY08 commitment is around SDR 11 million. The IDA 15 numbers for FY09-11 is still not available. 


\title{
AnNeX III. Tajikistan: Relations With The ASIAN Development Bank
}

\author{
(As of May 2008)
}

Country Director: Mr. Makoto Ojiro-Telephone: 992-372-210558/235314/235315

1. Tajikistan became a member of the Asian Development Bank (ADB) in 1998. The ADB has participated in the Consultative Group Meeting for Tajikistan since 1998. After conducting an initial mission in June 1998, ADB completed an Economic Report and Interim Operational Strategy that identified three areas: (a) agriculture; (b) infrastructure rehabilitation (especially energy and transport sectors); and (c) social sector, in which ADB assistance would have the greatest development impact. Based on the Interim Strategy, ADB's Board of Directors approved in October 1998 Tajikistan's country classification, which provides the basis for Tajikistan's full access to concessional resources (Asian Development Fund (ADF)). Under the new ADF grants framework, Tajikistan receives 50\% of its ADF allocation as loan and the remaining 50\% as grant, subject to a $20 \%$ volume discount. Under the performance-based allocation of ADF for 2007-2008, Tajikistan was allocated $\$ 63.24$ million for the two-year period. As of end 2007, Tajikistan has received $\$ 372.50$ million in loans, $\$ 33.00$ million in technical assistance, and $\$ 39.50$ million in grants.

2. In view of Tajikistan's urgent need for assistance in 1998, in coordination with the IMF and the World Bank (WB), the ADB provided a Postconflict Infrastructure Program Loan (\$20 million), which was designed to create a framework for developing market-based transport and energy sectors. The loan was for two years and was fully disbursed by end2000. Based on the satisfactory progress of the Postconflict Infrastructure Program loan conditionality, the following loans were approved in these two sectors:

3. Transport Sector: the Road Rehabilitation Project loan (\$20 million in 2000), which rehabilitated the most deteriorated sections of the Dushanbe-Kulyab road in the southern part of Tajikistan, the most civil-war affected areas; Dushanbe-Kyrgyz Border Road Rehabilitation Project loans (first phase of \$15 million in 2003 and second phase of \$30 million in 2005); and the Regional Road Corridor Improvement Project (\$53.4 million loan/grant in 2007), which improve Tajikistan's transportation link toward north and east through Kyrgyz Republic, further to Kazakhstan and Russia, as well as to the People's Republic of China. These projects also improve rural roads in the project areas. Continued supports in strengthening the government's institutional capacity for efficient management of national road network are provided under technical assistance grants associated with these loans and provided on stand-alone basis. In October 2007, ADB approved a \$2.0 million Japan Fund for Poverty Reduction grant to reconstruct key infrastructure in northeast Tajikistan to restore access to rural communities that were isolated after flooding in 2006 washed away a suspension bridge.

4. Power Sector: the Power Rehabilitation Project loan (\$34 million in 2000) aims to improve people's quality of life and support poverty reduction by increasing the availability of electricity, and assist in post-conflict recovery of Tajikistan's economy (the project rehabilitates and reinforces power transmission and distribution facilities in the war-damaged 
areas in Khatlon and Dushanbe regions, and rehabilitates the Nurek Power Plant and Central Hydropower Plant). The Regional Power Transmission Modernization Project loan (\$20 million in 2002) was geared towards improving the reliability and the operation of the Central Asian power transmission system, enhancing the intercountry power trading between Tajikistan and Uzbekistan, and laying the foundation for a future wholesale regional power market. However, the later loan had to be cancelled due to the inability of Tajikistan and Uzbekistan agreeing on the Power Trade Agreement. The Tajikistan-Afghanistan Power Transmission Interconnection Project loan for \$21.5 million to Tajikistan was approved in 2006. This project will construct a 220 kilovolt double circuit transmission line that will link the hydropower stations on Tajikistan's Vakhsh River to the border town of Sherkan Bandar, then to Kunduz, Baglad, Pul-e-Khumri and, ultimately, Kabul in Afghanistan. Various technical assistance projects were provided to accelerate market-oriented reforms in the power sector also in line with IMF and WB programs and for supporting the government in formulating a power sector development strategy. ADB is also providing a technical assistance to support the techno-economic assessment for the establishment of a Central Asia South Asia Regional Electricity Market (CASAREM) for electricity trading between Afghanistan, Kyrgyz Republic, Pakistan, and Tajikistan.

5. In addition to the above, the ADB Board approved to date the following loans to Tajikistan in each sector:

6. Social Sector: the Social Sector Rehabilitation Project loan (\$20 million in 1999) to address the serious deterioration in living standards and strengthen the delivery of essential social services; the Health Sector Reform Project loan (\$7.5 million in 2003), which aims to improve health, especially of the poor, women, and children by providing pro-poor health service package, and by reforming the health service delivery and financing mechanism; and the Education Sector Reform Project loan (\$7.5 million in 2003), which focuses on supporting the government's reform priorities in primary (grades 1-4) and general secondary education (grades 5-11) by improving the education system and its management. These two social sector reform loans are being supported by respective grants financed by Japan Fund for Poverty Reduction (JFPR): (a) the project for Community Participation and Public Information Campaign for Health Improvement (\$1 million in 2004); and (b) the School Improvement Project ( $\$ 2$ million in 2004), which aims to increase access to improved education for children, particularly girls, of poor families and vulnerable groups.

7. Agriculture Sector: based on the agriculture sector assessment prepared during 1999-2000, the Agriculture Rehabilitation Project loan (\$35 million in 2002) is being implemented to improve living conditions of the farming communities in the project area and to institute measures to sustain benefits of improvements for irrigation and drainage systems and water supply, as well as by providing farm production support services. In order to support the agriculture sector reforms, the TA for Farm Debt Resolution and Policy Reforms, which was associated with the loan, has been completed in consultation with the IMF, involving a wide range of stakeholders. Based on the findings of the TA, ADB supported the government in close cooperation with other donors and NGOs for formulating a National Farm Debt Resolution Strategy. A wide range of donor assistance is now needed as the Strategy has been announced by the government. ADB, in collaboration with DFID, is supporting donor coordination for implementing the Strategy. On 5 March 2007, the 
Government approved the Roadmap for Implementation of the Farm Debt Resolution Strategy. The road map, which includes a comprehensive program of reforms for the sustainable and profitable farming of cotton and other crops, guides the preparation of a suitable intervention for ADB. Grant assistance for Rural Poverty Reduction Project (\$2.9 million in 2001) financed by JFPR has supported the loan preparation and implementation by pilot testing innovative poverty-oriented on- and off-farm supports. In 2004, Irrigation Rehabilitation Project loan was approved for \$22.7 million. In a major push supporting the agriculture sector and rural development, Sustainable Cotton Sub-Sector Project loan/grant and Rural Development Project loan/grant were approved in 2006 and 2007, respectively.

8. Finance: Microfinance Systems Development Program loan ( $\$ 4$ million in 2003) to support policy, legal, and regulatory reforms; and Microfinance Systems Development Project loan (\$4 million in 2003) to help transform nongovernmental organization microfinance programs into licensed and regulated microfinance institutions.

9. Trade Facilitation: the Regional Trade Facilitation and Customs Cooperation Program loan (\$10 million in 2002), which supported trade and customs reform development across the East and Central Asia. Regional Customs Modernization and Infrastructure Development ( $\$ 10.7$ million in 2004) to promote international trade and enabling environment for private sector development.

10. Emergency Assistance: in response to the government's urgent requests, three emergency loans, including the Emergency Flood Rehabilitation Project loan ( $\$ 5$ million in 1999), the Emergency Restoration of Yavan Water Conveyance System loan (\$3.6 million in 2001), and the Emergency Baipaza Landslide Stabilization Project loan (\$5.3 million in 2002) were approved. In 2007, ADB provided a $\$ 22$ million loan for the Khatlon Province Flood Risk Management Project to help address recurring flood risks in four districts in the province through a comprehensive and coordinated approach. A major part of the project is the rehabilitation of 8.3 kilometers of flood protection embankment along Pyanj River, which borders Tajikistan and neighboring Afghanistan.

11. In end-2000, ADB started supporting the government in developing PRSP through participatory approach under a TA grant in close cooperation with the IMF, WB, and UNDP. Following the finalization of the PRSP in June 2002, ADB concluded the Poverty Partnership Agreement (PPA) with the government in December 2002, and proceeded to prepare a new five-year Country Strategy and Program (CSP) for 2004-08 for Tajikistan, which was endorsed by the ADB Board in October 2003. Its main objectives are (a) to strengthen rural development through institution building that will support policy implementation and the private sector; (b) to rehabilitate power and rural infrastructure; and (c) to strengthen regional cooperation through improved customs services and transport links, both within the country and neighboring ones.

12. ADB prepares and updates its three-year rolling programs for Tajikistan every year in consultation with the government based on the ADF resources availability, carefully examining the country's social and economic development status and in coordination with other donors, including IMF. The latest Country Operations Business Plan for Tajikistan covering 2008-2010 was approved in October 2007. Currently, a joint country support strategy 
13. The proposed 2008-2010 lending and grant program comprises three programs and three projects worth a total of $\$ 185.0$ million. The actual lending level will be determined by (a) availability of overall ADF resources; (b) country performance assessment vis-à-vis ADB's policy on performance based allocation of ADF resources; and (c) processing status of the projects in the pipeline. 


\section{AnNeX IV. TAJIKISTAN: Statistical IsSUES}

1. Tajikistan began participation in the GDDS in November 2004 and the country page in the International Financial Statistics (IFS) has been published since February 2003.

2. The authorities have indicated their interest in subscribing to the Fund's SDDS. They have appointed a national SDDS coordinator and requested technical assistance for this purpose. Before subscribing to the SDDS, Tajikistan will need to compile and disseminate the following datasets: quarterly national accounts, template on international reserves and foreign currency liquidity, and international investment position. Also, Tajikistan will need to disseminate regularly general government operations that include special funds (extrabudgetary accounts) at least on an annual basis, quarterly central government debt, monthly gross international reserves, and quarterly external debt data, with improved timeliness for several data categories.

3. The data ROSC mission in April 2004 found that the consumer price index, government finance statistics (GFS), and monetary statistics broadly follow sound methodologies. The mission also noted that source data are well-developed in all areas of statistics, and that suitable processes for assessment and validation of source data are in place. However, further efforts to improve the quality of economic statistics are limited by tight budgetary constraints.

\section{National Accounts and Price Statistics}

4. The ROSC mission identified significant deficiencies in statistical techniques for national accounts and price statistics, most notably in procedures to estimate the informal economy, and in the techniques for imputation, replacement, quality adjustment, and introduction of new products in the price indices. In addition, an intensified effort is needed to strengthen the scope, classification/sectorization, and basis of recording of the national accounts, and to reconsider the concepts and basis of recording underlying the producer price index (PPI).

\section{Fiscal Statistics}

5. The government finance statistics are based on cash transactions as recommended in A Manual on Government Finance Statistics, 1986. Although there are no plans at present to migrate the basis of compilation to the Government Finance Statistics Manual 2001, the cash GFS operations data were reported in the new framework for inclusion in the 2005 GFS Yearbook, although no details on financing of the cash deficit were reported. Data with a higher frequency for inclusion in the IFS are not reported. 


\section{Monetary and Financial Statistics}

6. A monetary and financial statistics (MFS) mission visited Tajikistan in early 2006 and assisted the authorities in establishing the procedures for compiling analytical accounts for commercial banks using data collected from the revised chart of accounts. The mission also assisted the authorities in implementing the Standardized Report Forms (SRFs) for MFS developed by STA. The authorities resumed regular reporting of monthly monetary data to STA for publication in IFS shortly after the mission. Further efforts are needed to implement the SRFs and the mission's other recommendations for improving the collection, compilation, and dissemination of MFS. However, due to the recent misreporting episode, the authorities stopped reporting monetary data to STA for publication in IFS since December 2007. The assessment of the adequacy of the data provision in this area is pending the special NBT audit.

\section{Balance of Payments Statistics}

7. There is a need for consistency in applying the residency concept in the balance of payments and the national accounts, and the scope of the foreign trade data needs to be improved to cover the shuttle trade. 
TAJIKISTAN: TABLE OF COMMON INDICATORS REQUIRED FOR SURVEILLANCE

(As of May 1, 2008)

\begin{tabular}{|c|c|c|c|c|c|c|c|}
\hline & $\begin{array}{l}\text { Date of latest } \\
\text { observation }\end{array}$ & $\begin{array}{l}\text { Date } \\
\text { received }\end{array}$ & $\begin{array}{l}\text { Frequency } \\
\text { of }\end{array}$ & $\begin{array}{l}\text { Frequency of } \\
\text { Renorting }\end{array}$ & $\begin{array}{l}\text { Frequency } \\
\text { of }\end{array}$ & \multicolumn{2}{|c|}{ Memo Items: } \\
\hline & & & & & & $\begin{array}{l}\text { Data Quality - } \\
\text { Methodological } \\
\text { soundness }\end{array}$ & $\begin{array}{l}\text { Data Quality - } \\
\text { Accuracy and } \\
\text { reliability }^{8}\end{array}$ \\
\hline Exchange Rates & 03/31/08 & 04/25/08 & D & M & W & & \\
\hline $\begin{array}{l}\text { International Reserve Assets and } \\
\text { Reserve Liabilities of the Monetary } \\
\text { Authorities }{ }^{1}\end{array}$ & 03/31/08 & 04/25/08 & w & M & W & & \\
\hline Reserve/Base Money & 03/31/08 & 04/25/08 & W & M & W & O, O, LO O & $\begin{array}{l}\text { LO, O, O, O, } \\
\text { NO }\end{array}$ \\
\hline Broad Money & 03/31/08 & 04/25/08 & M & M & M & & \\
\hline Central Bank Balance Sheet & 03/31/08 & 04/25/08 & W & M & W & & \\
\hline $\begin{array}{l}\text { Consolidated Balance Sheet of the } \\
\text { Banking System }\end{array}$ & 03/31/08 & 04/25/08 & M & M & M & & \\
\hline Interest Rates $^{2}$ & 03/31/08 & 04/25/08 & W & M & $\mathrm{v}$ & & \\
\hline Consumer Price Index & 03/31/08 & 04/25/08 & M & M & M & LO, LO, LNO, O & $\begin{array}{l}\text { LO, LO, LO, O, } \\
\text { O }\end{array}$ \\
\hline $\begin{array}{l}\text { Revenue, Expenditure, Balance } \\
\text { and Composition of Financing } \\
\text { General Government }^{4}\end{array}$ & 03/31/08 & $4 / 25 / 08$ & M & M & M & LO, LO, O, O & $\begin{array}{l}\text { O, O, O, LO, } \\
\text { LO }\end{array}$ \\
\hline $\begin{array}{l}\text { Revenue, Expenditure, Balance } \\
\text { and Composition of Financing }{ }^{3}- \\
\text { Central Government }\end{array}$ & 03/31/08 & $4 / 25 / 08$ & M & M & M & & \\
\hline $\begin{array}{l}\text { Stocks of Central Government and } \\
\text { Central Government-Guaranteed } \\
\text { Debt }\end{array}$ & Q3/08 & $4 / 25 / 08$ & Q & Q & $\mathrm{V}$ & & \\
\hline External Current Account Balance & Q3/08 & $4 / 25 / 08$ & Q & Q & $\mathrm{V}$ & $\begin{array}{l}\text { LNO, LNO, O, } \\
\text { O }\end{array}$ & LO, O, O, O, O \\
\hline $\begin{array}{l}\text { Exports and Imports of Goods and } \\
\text { Services }\end{array}$ & Q3/08 & $4 / 25 / 08$ & Q & Q & $\mathrm{v}$ & & \\
\hline GDP/GNP & 03/31/08 & $4 / 25 / 08$ & M & M & $\mathrm{V}$ & $\begin{array}{l}\text { O, LNO, LNO, } \\
\text { LNO }\end{array}$ & $\begin{array}{l}\text { LO, LO, LNO, } \\
\text { O, O }\end{array}$ \\
\hline Gross External Debt ${ }^{5}$ & Q3/08 & $4 / 25 / 08$ & Q & Q & $\mathrm{V}$ & & \\
\hline
\end{tabular}

${ }^{1}$ Includes reserve assets pledged or otherwise encumbered, as well as net derivative positions.

${ }^{2}$ Both market-based and officially determined, including discount rates, money market rates, and rates on treasury bills, notes and bonds.

${ }^{3}$ Foreign, domestic bank, and domestic nonbank financing.

${ }^{4}$ The general government comprises central government (budgetary, extra budgetary, and social protection funds) and state and local governments.

${ }^{5}$ Including currency and maturity composition.

${ }^{6}$ Daily (D), Weekly (W), Monthly (M), Quarterly (Q), Annually (A); NA: Not Available.

${ }^{7}$ Reflects the assessment provided in the data ROSC published in April 2005 and based on the findings of the staff mission during April 2004 for the dataset corresponding to the variable in each row. The assessment indicates whether international standards concerning concepts and definitions, scope, classification/sectorization, and basis for recording are fully observed (O), largely observed (LO), largely not observed (LNO), or not observed (NO).

${ }^{8}$ Same as footnote 7, except referring to international standards concerning source data, statistical techniques, assessment and validation of source data, assessment and validation of intermediate data and statistical outputs, and revision studies. 